Notfall Rettungsmed $2017 \cdot 20$ (Suppl 1):S3-S24 DOI 10.1007/s10049-017-0328-0

Online publiziert: 29. Juni 2017

(c) European Resuscitation Council (ERC), German Resuscitation Council (GRC), Austrian Resuscitation Council (ARC) 2017

CrossMark

G.D. Perkins ${ }^{1,2} \cdot$ A.J. Handley ${ }^{3}$ R.W. Koster ${ }^{4}$ M. Castrén ${ }^{5} \cdot$ M.A. Smyth ${ }^{1,6} \cdot$ T. Olasveengen ${ }^{7} \cdot$ K.G. Monsieurs ${ }^{8,9} \cdot$ V. Raffay ${ }^{10} \cdot$ J.-T. Gräsner ${ }^{11} \cdot$ V. Wenzel ${ }^{12} \cdot$ G. Ristagno ${ }^{13} \cdot$ J. Soar ${ }^{14}$

'Warwick Medical School, University of Warwick, Coventry, UK; ${ }^{2}$ Critical Care Unit, Heart of England NHS Foundation Trust, Birmingham, UK; ${ }^{3}$ Hadstock, Cambridge, UK; ${ }^{4}$ Department of Cardiology, Academic Medical Center, Amsterdam, Niederlande; ${ }^{5}$ Department of Emergency Medicine and Services, Helsinki University Hospital and Helsinki University, Helsinki, Finnland; ${ }^{6}$ West Midlands Ambulance Service NHS Foundation Trust, Dudley, UK; ${ }^{7}$ Norwegian National Advisory Unit on Prehospital Emergency Medicine and Department of Anesthesiology, Oslo University Hospital, Oslo, Norwegen; ${ }^{8}$ Emergency Medicine, Faculty of Medicine and Health Sciences, University of Antwerp, Antwerp, Belgien; ${ }^{9}$ Faculty of Medicine and Health Sciences, University of Ghent, Ghent, Belgien; ${ }^{10}$ Municipal Institute for Emergency Medicine Novi Sad, Novi Sad, Serbien; "Department of Anaesthesia and Intensive Care Medicine, University Medical Center Schleswig-Holstein, Kiel, Deutschland; ${ }^{12}$ Department of Anesthesiology and Critical Care Medicine, Medical University of Innsbruck, Innsbruck, Österreich; ${ }^{13}$ Department of Cardiovascular Research, IRCCS-Istituto di Ricerche Farmacologiche "Mario Negri", Milan, Italien; ${ }^{14}$ Anaesthesia and Intensive Care Medicine, Southmead Hospital, Bristol, UK

\title{
Basismaßnahmen zur
} Wiederbelebung Erwachsener und Verwendung automatisierter externer Defibrillatoren

\section{Kapitel 2 der Leitlinien zur Reanimation 2015 des European Resuscitation Council}

anfälle bei Atemwegsverlegung durch Fremdkörper beherrscht werden können. Leitlinien für den Einsatz manueller Defibrillatoren und zur Einleitung von Wiederbelebungsmaßnahmen im Krankenhaus finden sich in Kap. 3 (ALS) [1]. Eine Kurzdarstellung der Seitenlage ist enthalten; detaillierter wird auf diese in Kap. 9 (EH) eingegangen [2].

Die Leitlinien zu BLS/AED [3] basieren auf dem ILCOR 2015 Consensus on Science and Treatment Recommendations (CoSTR). Der ILCOR Review behandelt 23 Themen und führt zu 32 Behandlungsempfehlungen zu den Themen: frühes Erkennen der Situation und Verhindern eines Kreislaufstillstands, frühe hochwertige Herzdruckmassage (Thoraxkompression) und frühe Defibrillation.

Für die ERC-Leitlinien wurde zusätzlich zu den ILCOR-Empfehlungen von den federführenden Autoren die Literatur zu den Themenfeldern gesichtet, die bei ILCOR keine Berücksichtigung fanden. Die Leitlinien-Verfasser waren sich bewusst, dass Änderungen gegenüber den Empfehlungen von 2010 Kosten und eventuell Verunsicherung verursachen würden, und beschränkten sich daher auf Änderungen, die wirklich wichtig erschienen und durch neue Evidenz belegt sind. Die Leitlinien wurden von den federführenden Autoren konzipiert, von allen Leitlinien-Verfassern und den nationalen Wiederbelebungsräten überarbeitet und schließlich vom ERC-Board verabschiedet.

\section{Zusammenfassung der Änderungen}

Die Leitlinien 2015 betonen die besondere Bedeutung der Interaktion des Leitstellendisponenten mit dem Notfallzeugen, der mit der Wiederbelebung beginnt, und der zeitnahen Verfügbarkeit eines Defibrillators. Eine effektive, koordinierte des originalen Beitrags: 10.1007/s10049-015$0081-1$. 


\section{Zu diesem Kapitel haben beigetragen:}

Leo L Bossaert: University of Antwerp

Antwerp, Belgium

Antonio Caballero: Emergency Department, Hospital Universitario Virgen del Rocío, Sevilla, Spain

Pascal Cassan: Global First Aid Reference Centre, International Federation of Red Cross and Red Crescent, Paris, France

Cristina Granja: Emergency and Intensive Care Department, Hospital de Faro, Centro Hospitalar do Algarve, Porto, Portugal

Claudio Sandroni: Department of Anaesthesiology and Intensive Care, Catholic University School of Medicine, Rome, Italy

David A Zideman: Imperial College Healthcare NHS Trust, London, UK

Jerry P. Nolan: Department of Anaesthesia and Intensive Care Medicine, Royal United Hospital, Bath, UK

Ian Maconachie: Paediatric Emergency Medicine and NIHR BRC, Imperial College, London, UK

Robert Greif: Department of Anaesthesiology and Pain Medicine, University Hospital Bern and University Bern, Bern, Switzerland

gemeinsame Aktion, die diese Elemente zusammenführt, verbessert die Überlebenschancen des Patienten nach einem Kreislaufstillstand außerhalb eines Krankenhauses (• Abb. 1).

Der Leitstellendisponent spielt eine entscheidende Rolle bei der frühzeitigen Diagnose eines Kreislaufstillstands, der leitstellengeführten Reanimation (bekannt als Telefonreanimation) und bei Auffinden und Einsetzen eines externen automatisierten Defibrillators (AED). Je früher der Rettungsdienst alarmiert wird, desto eher wird eine adäquate Behandlung begonnen und unterstützt.

Fertigkeiten, Zutrauen und Handlungsbereitschaft des Ersthelfers hängen von den Umständen des Kreislaufstillstands, seinem Trainingsstand und seinen Vorkenntnissen ab.

Der ERC empfiehlt dem Notfallzeugen, der darin geschult und dazu in der Lage ist, den Zustand des Patienten schnell zu beurteilen, indem er feststellt,

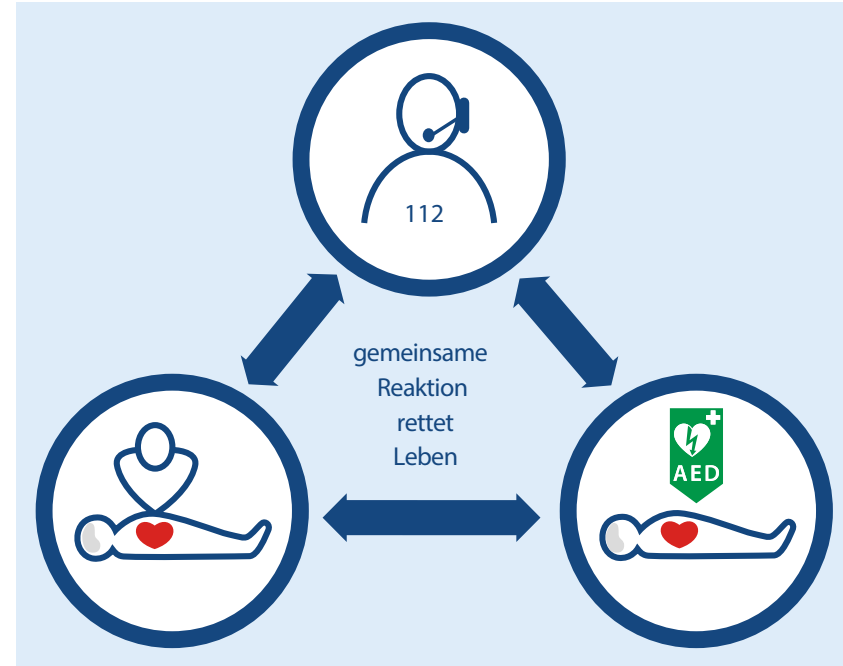

Abb. $1<$ Interaktion zwischen Leitstellendisponent, Helfer und Einsatz eines automatisierten externen Defibrillators

ob der Patient nicht ansprechbar ist und nicht normal atmet, und dann unmittelbar den Rettungsdienst zu alarmieren.

Wenn irgend möglich, soll er währenddessen den Patienten nicht verlassen.

Der Patient, der nicht reagiert und nicht normal atmet, hat einen Kreislaufstillstand und benötigt eine Herz-Lungen-Wiederbelebung (CPR). Unmittelbar nach dem Kreislaufstillstand geht der Blutfluss zum Gehirn gegen null. Das kann Krampfanfälle auslösen, die möglicherweise mit einer Epilepsie verwechselt werden. Notfallzeugen und Leitstellendisponenten sollen daher bei krampfenden Patienten auch an einen Kreislaufstillstand denken und sorgfältig klären, ob der Patient normal atmet.

Die Leitlinien-Verfasser bekräftigen die ILCOR-Empfehlung, dass bei jeder Wiederbelebung eine Thoraxkompression durchgeführt werden soll. Notfallzeugen, die ausgebildet und in der Lage sind, eine Atemspende durchzuführen, sollen Herzdruckmassage und Atemspende kombinieren. Zusätzliche Atemspenden können vorteilhaft für Kinder bei asphyktischem Kreislaufstillstand sein oder wenn sich das Eintreffen des Rettungsdienstes verzögert.

Da wir nicht davon überzeugt sind, dass eine Wiederbelebung allein durch Thoraxkompressionen einer Standardwiederbelebung gleichwertig ist, empfehlen wir weiterhin die bisher praktizierte Vorgehensweise.
Eine qualitativ hochwertige Wiederbelebung ist entscheidend für eine Verbesserung des Ergebnisses (Outcome). Bei der Herzdruckmassage soll eine adäquate Drucktiefe sicher erreicht werden (etwa $5 \mathrm{~cm}$, jedoch nicht mehr als $6 \mathrm{~cm}$ beim normalen Erwachsenen), bei einer Kompressionsfrequenz von 100-120/min mit minimalen Unterbrechungen. Nach jeder Kompression muss der Brustkorb vollständig entlastet werden. Atemspenden/Beatmungen sollen eine Sekunde dauern und zu einer deutlichen Hebung des Brustkorbs führen. Das Verhältnis von Herzdruckmassage zu Beatmung bleibt 30:2. Unterbrechen Sie die Thoraxkompressionen für die Beatmung nicht länger als $10 \mathrm{~s}$.

Durch Defibrillation innerhalb von 3-5 min nach dem Kollaps können Überlebensraten von $50-70 \%$ erreicht werden. Eine frühzeitige Defibrillation kann durch Notfallzeugen unter Verwendung von öffentlichen oder hauseigenen AEDs durchgeführt werden. Public-Access-Programme (öffentlicher Zugang zu AEDs) sollen an viel besuchten Orten (Flughäfen, Bahn- oder Busstationen, Sportstätten, Einkaufszentren, Bürogebäuden und Kasinos) etabliert werden. Kreislaufstillstände an solchen Orten werden häufig beobachtet, und ausgebildete Helfer können schnell vor Ort sein. Das Vorhalten eines AED gilt schon dann als kosteneffektiv, wenn sich an seinem Standort alle 5 Jahre ein Kreislaufstillstand ereignet, die Kosten pro gewonnenes Lebensjahr entsprechen denen 


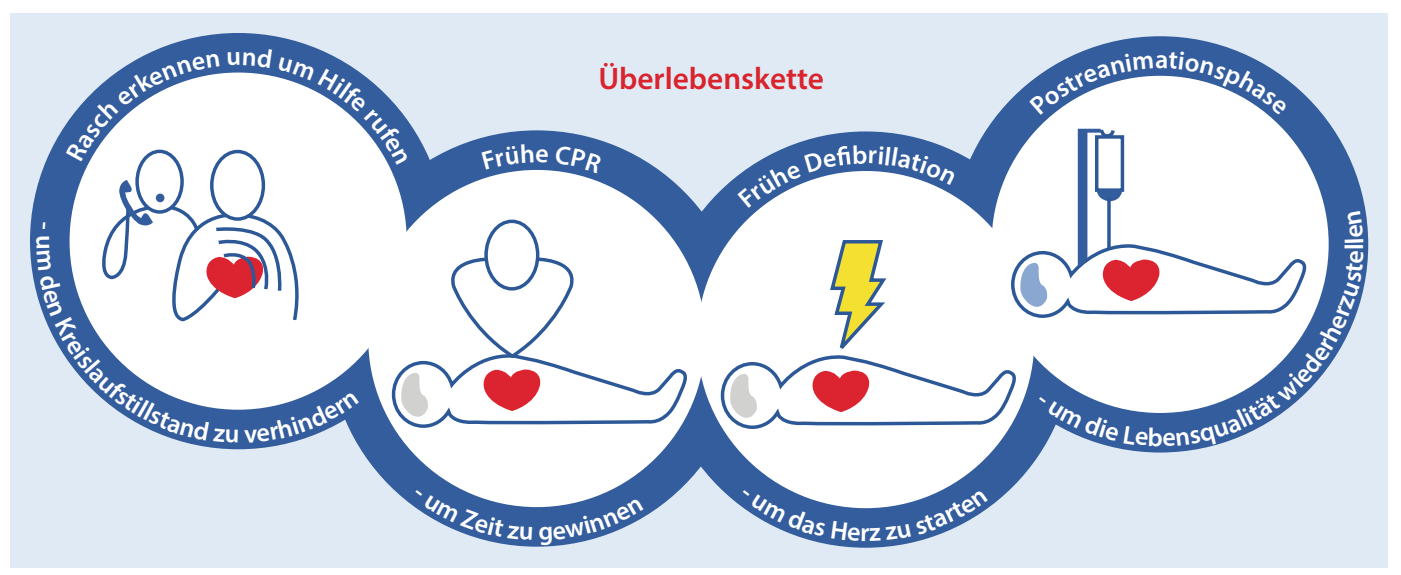

Abb. $\mathbf{2}<$ Die Überlebenskette

anderer medizinischer Interventionen. Erfahrungen mit Kreislaufstillständen in der Vergangenheit und die Art der Umgebung können bei der Wahl der AED-Platzierung wegweisend sein. Eine Registrierung beim Rettungsdienst ermöglicht es den Disponenten, Helfer zu einem nahe gelegenen AED zu führen und so die Reaktionszeit zu verbessern.

Der Ablauf der Wiederbelebungsmaßnahmen für Erwachsene kann auch bei Kindern, die nicht ansprechbar sind und nicht normal atmen, sicher verwendet werden. Helfer mit einer entsprechenden Ausbildung können bei Kindern die Wiederbelebungschance mit 5 initialen Beatmungen verbessern. Für den eher seltenen Fall, dass ein Helfer auf sich allein gestellt ist, ist es bei Kindern und Ertrunkenen auch besser, erst aktiv zu werden und verzögert Hilfe zu holen. Thoraxkompressionen bei Kindern sollten eine Tiefe von mindestens einem Drittel der Brustkorbhöhe haben (Kleinkinder $4 \mathrm{~cm}$, Schulkinder $5 \mathrm{~cm}$ ).

Die Verlegung der Atemwege durch einen Fremdkörper ist ein medizinischer Notfall. Diese tritt meistens beim Essen und Trinken ein und erfordert sofortiges Eingreifen durch Schläge auf den Rücken. Führt dies nicht zum Erfolg, sind Oberbauchkompressionen erforderlich. Reagiert der Patient nicht mehr, müssen Wiederbelebungsmaßnahmen durchgeführt und Hilfe herbeigerufen werden.

\section{Kreislaufstillstand}

Der plötzliche Kreislaufstillstand stellt eine der Haupttodesursachen in Europa dar. Je nachdem, wie der plötzliche
Kreislaufstillstand definiert wird, betrifft er zwischen 350.000 und 700.000 Menschen pro Jahr [4-6]. Zum Zeitpunkt der ersten Analyse des Herzrhythmus weisen 25-50\% der Betroffenen Kammerflimmern („ventricular fibrillation“, VF) auf; dieser Anteil hat sich in den letzten 20 Jahren verringert [7-13]. Wahrscheinlich haben zum Zeitpunkt des Kollapses weitaus mehr Patienten Kammerflimmern oder eine schnelle Kammertachykardie („ventrikuläre Tachykardie“, VT), aber bis zur Aufzeichnung des ersten Elektrokardiogramms (EKG) durch Rettungsdienstpersonal hat sich der Rhythmus zur Asystolie verschlechtert $[14,15]$. Wird der Herzrhythmus kurz nach dem Kollaps aufgezeichnet, insbesondere durch einen vor Ort verfügbaren AED, steigt der Anteil der Patienten mit Kammerflimmern auf bis $\mathrm{zu} 76 \%$ [16, 17]. Viele dieser Patienten überleben, wenn Notfallzeugen schon handeln, während noch Kammerflimmern vorliegt. Dagegen ist eine erfolgreiche Wiederbelebung unwahrscheinlich, wenn sich der Herzrhythmus bereits zu einer Asystolie verschlechtert hat.

Die empfohlene Behandlung bei einem VF-Kreislaufstillstand ist die sofortige Wiederbelebung durch frühzeitige elektrische Defibrillation. Den meisten Fällen von nichtkardialem Kreislaufstillstand liegt eine Störung der Atemfunktion zugrunde, wie Ertrinken (häufig Kinder) und Asphyxie. Beatmung und Thoraxkompressionen sind bei diesen Patienten für eine erfolgreiche Wiederbelebung entscheidend.

\section{Die Überlebenskette}

Die Überlebenskette fasst die für eine erfolgreiche Wiederbelebung entscheidenden Schritte zusammen (• Abb. 2). Die meisten Kettenglieder treffen für Patienten mit primär kardial bedingtem wie auch primär asphyktisch bedingtem Kreislaufstillstand zu [18].

\section{1 - Frühes Erkennen und Notruf}

Brustschmerzen müssen zunächst als Mangeldurchblutung des Herzmuskels erkannt werden. Ein Viertel bis ein Drittel solcher Patienten erleidet in der ersten Stunde nach Schmerzbeginn einen Kreislaufstillstand [19]. Erkennt man, dass der Schmerz herzbedingt ist und ruft den Rettungsdienst, bevor der Patient kollabiert, wird dieser früher eintreffen, und zwar hoffentlich, bevor der Kreislaufstillstand eintritt, sodass die Überlebenschancen verbessert werden [20-23].

Ist der Kreislaufstillstand eingetreten, so kommt es darauf an, ihn schnell zu erkennen, um umgehend den Rettungsdienst zu rufen und sofort mit ErsthelferReanimation zu beginnen. Die Schlüsselsymptome sind fehlende Reaktion und nicht normale Atmung. Leitstellendisponenten können die Diagnose beschleunigen, indem sie sich auf das Abfragen dieser Schlüsselsymptome beschränken.

\section{2 - Frühe Wiederbelebung durch Notfallzeugen}

Unverzüglich eingeleitete Wiederbelebungsmaßnahmen können die Überle- 
bensrate bei Kreislaufstillstand verdoppeln bis vervierfachen [20, 24-28]. Ein ausgebildeter Helfer soll Thoraxkompressionen und Beatmung kombinieren. Ist ein Anrufer nicht in Wiederbelebung ausgebildet, soll der Leitstellendisponent ihn oder sie anleiten, ausschließlich Herzdruckmassage durchzuführen, bis professionelle Hilfe eintrifft [29-31].

\section{3 - Frühe Defibrillation}

Defibrillation innerhalb von 3-5 min nach dem Kollaps kann die Überlebensrate auf $50-70 \%$ erhöhen. Dies kann durch öffentlich zugängliche und hauseigene AEDs erreicht werden [13, 17, 32, 33]. Jede Minute Verzögerung vor der Defibrillation vermindert die Wahrscheinlichkeit des Überlebens bis zur Klinikentlassung um 10-12\%. Die Glieder der Kette zusammen greifen besser: Wenn Notfallzeugen mit einer Wiederbelebung beginnen, sinken die Überlebenschancen langsamer, pro Minute Verzögerung im Mittel um 3-4\% $[20,24,34]$.

\section{4 - Frühe erweiterte Maßnahmen und standardisierte Behandlung nach der Reanimation}

Erweiterte Maßnahmen wie Atemwegsmanagement, Medikamentengabe und Behandlung der Ursachen können erforderlich sein, wenn die Wiederbelebungsmaßnahmen primär nicht erfolgreich sind. Die Qualität der Behandlung in der Postreanimationsphase beeinflusst das Ergebnis, sie werden in den Kapiteln „erweiterte Maßnahmen“ und „Postreanimationsbehandlung" behandelt $[1$, 35].

\section{Notfallzeugen müssen sofort handeln}

In den meisten Gemeinden liegt die durchschnittliche Zeitspanne zwischen der Alarmierung und dem Eintreffen des Rettungsdienstes (Hilfsfrist) bei 5-8 min $[16,36-38]$ bzw. $8-11$ min bis zum ersten Defibrillationsschock [13, 27]. Während dieser Zeit hängt das Überleben des Patienten davon ab, dass Notfallzeugen mit der Wiederbelebung beginnen und einen AED einsetzen.

Opfer eines Kreislaufstillstands benötigen sofortige Wiederbelebungsmaßnahmen. Diese erzeugen einen geringen, aber entscheidenden Blutfluss zum Herzen und zum Gehirn. Außerdem steigern sie die Wahrscheinlichkeit, dass das Herz wieder einen effektiven Rhythmus und eine effektive Pumpleistung aufnimmt. Thoraxkompressionen sind besonders wichtig, wenn nicht in den ersten Minuten nach dem Kollaps ein Schock verabreicht werden kann [39]. Wenn das Herz noch lebensfähig ist, nehmen seine normalen Schrittmacherzellen nach der Defibrillation ihre Funktion wieder auf und produzieren einen organisierten Rhythmus, dem mechanische Kontraktionen folgen. In den ersten Minuten nach einer erfolgreichen Beendigung des Kammerflimmerns können der Herzrhythmus langsam und die Kraft der Kontraktionen schwach sein; die Thoraxkompressionen müssen daher fortgesetzt werden, bis eine ausreichende Herzfunktion zurückkehrt.

Die Verwendung eines AED durch Laienhelfer kann die Überlebenschance bei einem Kreislaufstillstand im öffentlichen Raum verbessern [16]. AEDs werden auch zunehmend in Wohnbereichen vorgehalten [40]. Ein AED gibt Sprachanweisungen, die den Ersthelfer anleiten, er analysiert den Herzrhythmus des Patienten und fordert den Ersthelfer auf, einen Schock abzugeben, wenn VF oder eine VT erkannt wird. AEDs sind genau und geben nur dann einen Schock ab, wenn VF (oder schnelle VT) vorliegt [41, 42].

\section{Erkennen des Kreislauf- stillstands}

Das Erkennen eines Kreislaufstillstands kann eine Herausforderung darstellen. Notfallzeuge und Leitstellendisponent müssen beide rasch die Diagnose stellen, um die Überlebenskette zu aktivieren. Die Prüfung des Karotispulses (oder eines anderen Pulses) hat sich für Laienwie auch für professionelle Helfer als ungenaue Methode heraugestellt, um festzustellen, ob ein Kreislauf vorhanden ist [43-47].
Schnappatmung ist eine langsame und tiefe Atmung, oft als charakteristisch schnarchendes Geräusch $\mathrm{zu}$ hören. Sie wird vom Hirnstamm gesteuert, dem Teil des Gehirns, der auch bei Sauerstoffmangel noch einige Minuten funktionsfähig bleibt. Das Vorhandensein von Schnappatmung kann irrtümlich als Beweis interpretiert werden, dass ein Kreislauf vorhanden und keine Wiederbelebung erforderlich ist. Schnappatmung kann bei Kreislaufstillstand bei bis zu $40 \%$ der Betroffenen auftreten. Wenn das als Zeichen für Kreislaufstillstand interpretiert und entsprechend reagiert wird, führt das $\mathrm{zu}$ größeren Überlebenschancen. Die Bedeutung von Schnappatmung soll daher bei der Ausbildung in Wiederbelebung hervorgehoben werden $[49,50]$. Notfallzeugen sollen einen Kreislaufstillstand annehmen und mit Thoraxkompressionen beginnen, wenn der Patient nicht reagiert und nicht normal atmet.

Unmittelbar nach einem Kreislaufstillstand geht der Blutzufluss zum Gehirn gegen null, als Folge können Krämpfe auftreten, die nicht mit einer Epilepsie verwechselt werden dürfen. Notfallzeugen sollen an einen Kreislaufstillstand denken, wenn sie einen krampfenden Patienten vorfinden [51, 52]. Von Notfallzeugen beobachtete Hautveränderungen wie Blässe oder die Blauverfärbung der Haut (Zyanose) können nicht als diagnostische Zeichen des Kreislaufstillstands gelten [51].

\section{Die Rolle des Leitstellen- disponenten}

Der Leitstellendisponent spielt eine entscheidende Rolle bei der Diagnose eines Kreislaufstillstands, beider Anleitung zur Reanimation (Telefonreanimation), bei Hinweisen zum Standort und Herbeiholen eines AED und bei der vordringlichen Alarmierung des Rettungsdienstes. Je früher der professionelle Rettungsdienst alarmiert wird, desto eher kann die erforderliche Behandlung in Gang gebracht und unterstützt werden. 


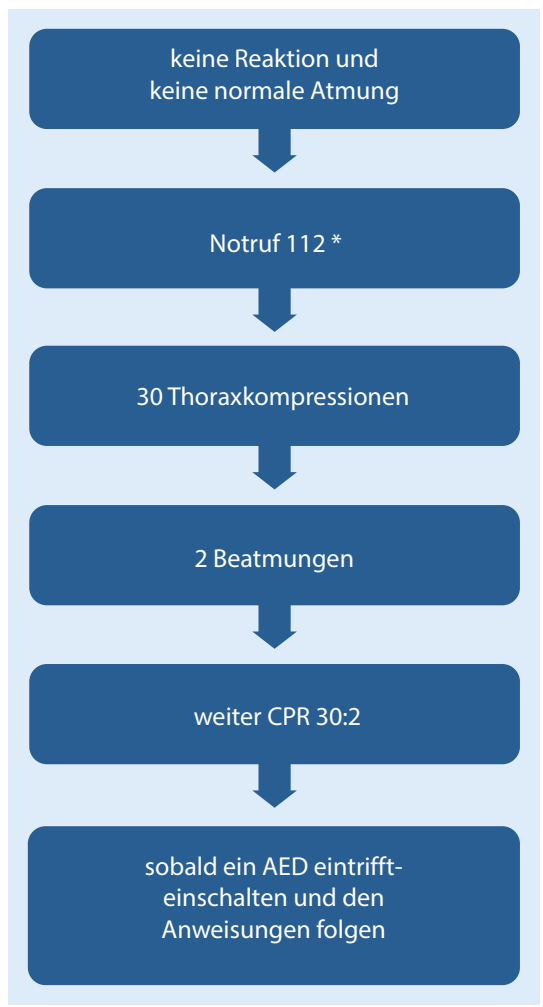

Abb. 3 ॥ Handlungsablauf zur Wiederbelebung Erwachsener. * Österreich/Schweiz 144

\section{Erkennen des Kreislaufstillstands durch den Disponenten}

Das Erkennen eines Kreislaufstillstands zum frühestmöglichen Zeitpunkt ist wichtig. Erkennt der Disponent den Kreislaufstillstand, ist das Überleben wahrscheinlicher, weil die richtigen Maßnahmen ergriffen werden können [53, 54]. Die Verbesserung der Fähigkeit des Disponenten, einen Kreislaufstillstand zu erkennen und den Dispositionsablauf zu optimieren, kann eine kostengünstige Lösung sein, um das Outcome nach Kreislaufstillstand zu verbessern.

Schriftliche Dispositionsprotokolle in den Leitstellen mit spezifischen Fragen zum schnelleren Erkennen eines Kreislaufstillstands können hilfreich sein. Bei Patienten, die nicht reagieren und nicht normal atmen, muss ein Kreislaufstillstand angenommen werden. Das Befolgen solcher Protokolle kann das Erkennen eines Kreislaufstillstands verbessern [9, 55-57], während solche Protokolle nicht zu befolgen das Erkennen des Kreislaufstillstands verzögert und die Anlei- tung zur Telefonreanimation durch den Disponenten verhindert [56-60].

Eine genaue Beschreibung der Atmung eines Patienten zu erhalten stellt für den Leitstellendisponenten eine Herausforderung dar. Eine agonale Atmung ist häufig, und Anrufer können fälschlicherweise glauben, der Patient atme normal [9, 60-68]. Ergänzende Schulung der Disponenten speziell zum Erkennen und zur Bedeutung der agonalen Atmung kann die Diagnose des Kreislaufstillstands beschleunigen, die Versorgung mit Telefonreanimation verbessern und die Zahl übersehener Kreislaufstillstände verringern [64].

Fragen zum Atemmuster und der Regelmäßigkeit der Atmung können helfen, eine anormale Atmung zu erkennen und so den Kreislaufstillstand zu identifizieren. Wenn beim Notruf von einer krampfenden Person berichtet wird, muss der Disponent den starken Verdacht haben, dass ein Kreislaufstillstand vorliegt, selbst wenn aus der Vorgeschichte des Patienten eine Epilepsie bekannt ist $[61,69]$.

\section{Leitstellengeleitete Reanimation}

Wiederbelebung durch Notfallzeugen ist nach wie vor selten. Es konnte jedoch gezeigt werden, dass sie durch Leitstellenassistenz (Telefonreanimation) häufiger wird [9, 56, 70-72], die Zeit des therapiefreien Intervalls sich verkürzt [56, 57, 68, 72, 73], die Anzahl der Thoraxkompressionen erhöht wird [70] und das Outcome der Patienten nach Kreislaufstillstand außerhalb des Krankenhauses (OHCA) über alle untersuchten Patientengruppen hinweg verbessert werden kann $[9,29-31,57,71,74]$.

Leitstellendisponenten sollen bei dem Verdacht auf einen Kreislaufstillstand immer eine Telefonreanimation anbieten, wenn kein trainierter Ersthelfer vor Ort ist. Handelt es sich um einen erwachsenen Patienten, soll der Disponent ausschließlich Instruktionen zur Thoraxkompression geben.

Bei einem kindlichen Notfall soll der Disponent Anleitung zur Beatmung und Thoraxkompression geben. Leitstellendisponenten sollen dementsprechend geschult sein, zu beiden Techniken anzuleiten.

\section{BLS-Ablauf beim Erwachsenen}

Alle Schritte zur initialen Beurteilung und zur Behandlung eines nicht reagierenden Patienten sind in $\mathbf{A b b} \mathbf{3}$ wiedergegeben. Sie führt den Leser vom Erkennen eines Kreislaufstillstands über die Alarmierung des Rettungsdienstes zum Start der Wiederbelebung und zum Einsatz des AED. Die Zahl der Schritte wurde auf die Schlüsselaktionen reduziert. Der überarbeitete Algorithmus soll schlüssig und logisch sein und es Helfern jeder Art ermöglichen, die Inhalte zu lernen, zu erinnern und anzuwenden.

- Abb. 3 zeigt Schritt für Schritt das Vorgehen für den trainierten Helfer, es legt weiterhin großen Wert darauf, ihm, dem Helfer, Patienten und Notfallzeugen Sicherheit zu geben. Der Ruf nach zusätzlicher Unterstützung (sofern nötig) ist in den Schritt „Notruf“ unten eingebettet. Der besseren Übersicht halber ist der Algorithmus linear dargestellt. Er ist so zu verstehen, dass die ersten Schritte - Reaktionsfähigkeit überprüfen, Atemwege öffnen, Atemkontrolle und Notruf - simultan oder in rascher Folge abgearbeitet werden können.

Wer nicht gelernt hat, einen Kreislaufstillstand zu identifizieren und mit einer Wiederbelebung zu beginnen, wird auch diese Leitlinien nicht kennen und die Hilfe des Disponenten benötigen, wenn er den Notruf 112 absetzt. Diese Leitlinie enthält deshalb keine speziellen Empfehlungen für diesen Personenkreis.

Im folgenden Teil dieses Kapitels werden Zusatzinformationen zu einigen Schritten des Gesamtablaufs gegeben.

\section{Freimachen der Atemwege und Atemkontrolle}

Der trainierte Helfer soll den kollabierten Patienten schnell beurteilen, um festzustellen, ob er reagiert und normal atmet.

Öffnen Sie die Atemwege durch Überstrecken des Nackens und Anheben des Kinns, und beurteilen Sie dabei, ob die Person normal atmet. Verzögern Sie die Beurteilung nicht, um die Atemwege auf Behinderungen zu überprüfen. Das Vorschieben des Unterkiefers (EsmarchHeiberg-Handgriff) und Auswischen des Mundes wird für den Laienhelfer 


\section{ERC Leitlinien}

\section{Vergewissern Sie sich, dass der Kollabierte und Notfallzeugen nicht gefährdet sind}

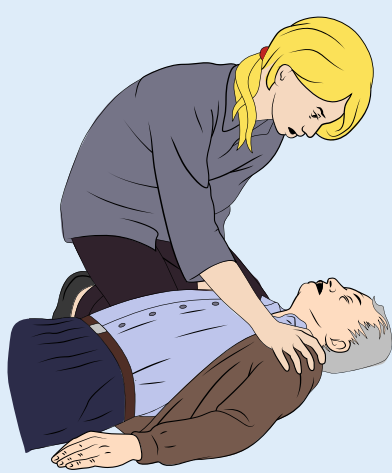

Reaktion: Prüfen Sie ob die Person reagiert

Schütteln Sie ihn leicht an den Schultern und fragen Sie laut: „Ist alles in Ordnung?"

Wenn er reagiert lassen Sie ihn, wenn keine weitere Gefahr besteht, in der Lage, in der Sie ihn vorgefunden haben.

Versuchen Sie herauszufinden, was mit ihm los ist, und holen Sie falls erforderlich Hilfe. Überprüfen Sie regelmäßig seinen Zustand.

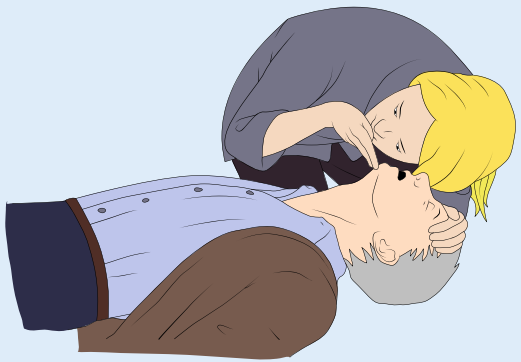

Atmung:

Kontrollieren Sie die Atmung durch Sehen, Hören und Fühlen Während der ersten Minuten nach einem Kreislauf-Stillstand ist es möglich, dass ein Patient kaum atmet oder nur vereinzelte, langsame oder geräuschvolle Atemzüge macht.

Verwechseln Sie dies nicht mit normaler Atmung. Sehen, hören und fühlen Sie nicht länger als $10 \mathrm{~s}$, um festzustellen, ob der Patient normal atmet.

Wenn Sie irgendwelche Zweifel haben, ob die Atmung norma ist, dann handeln Sie so, als sei sie nicht normal und beginnen mit CPR.

Reagiert der Patient nicht und atmet er nicht normal: Alarmieren Sie den Rettungsdienstes

Wenn möglich bitten sie jemanden den Rettungsdienst anzurufen (112) sonst rufen Sie selbst an

Verlassen Sie den Patienten nur, wenn es keine andere Möglichkeit gibt.

Schalten Sie Ihr Telefon auf „Freisprechen“ um leichte mit dem Leitstellendisponenten sprechen zu können.

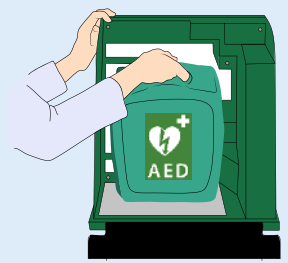

AED: Lassen Sie einen AED holen

Schicken sie jemand los, einen AED zu holen. Sind

Sie allein, verlassen Sie den Patienten nicht

beginnen Sie CPR

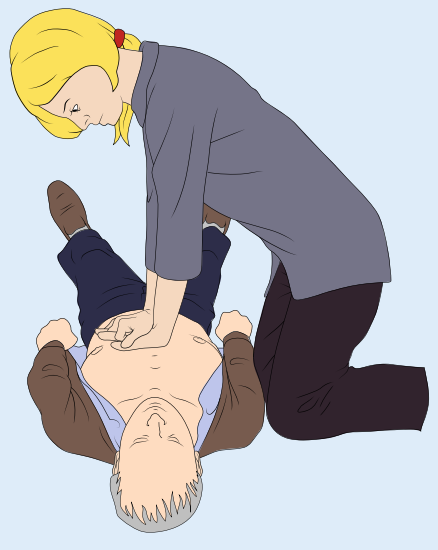

Kreislauf: Beginnen Sie mit Thoraxkompressionen Knien Sie sich neben den Patienten.

Legen Sie den Ballen einer Hand auf die Mitte der Brust des Patienten (entspricht der unteren Hälfte des Brustbeins [Sternum]).

Legen Sie den Ballen Ihrer anderen Hand auf die erste Hand.

Verschränken Sie die Finger Ihrer Hände ineinander und vergewissern Sie sich, sie nicht auf die Rippen des Patienten drücken.

Halten Sie die Arme gerade.

Üben Sie keinerlei Druck auf den Oberbauch oder das untere Ende des Brustbeins aus.

Bringen Sie ihre Schultern senkrecht über den Brustkorb des

Patienten, und drücken Sie das Brustbein mindestens $5 \mathrm{~cm}$

(jedoch nicht mehr als $6 \mathrm{~cm}$ ) nach unten.

Entlasten Sie nach jeder Kompression vollständig den

Brustkorb, ohne den Kontakt zwischen Ihren Händen und dem

Brustbein zu verlieren.

Wiederholen Sie dies mit einer Frequenz von 100-120/min

Abb. 4 \& Schritt-für-Schritt-Erklärung zur Wiederbelebung Erwachsener mit Kreislaufstillstand für den ausgebildeten BLS-AED-Helfer (Teil 1) 


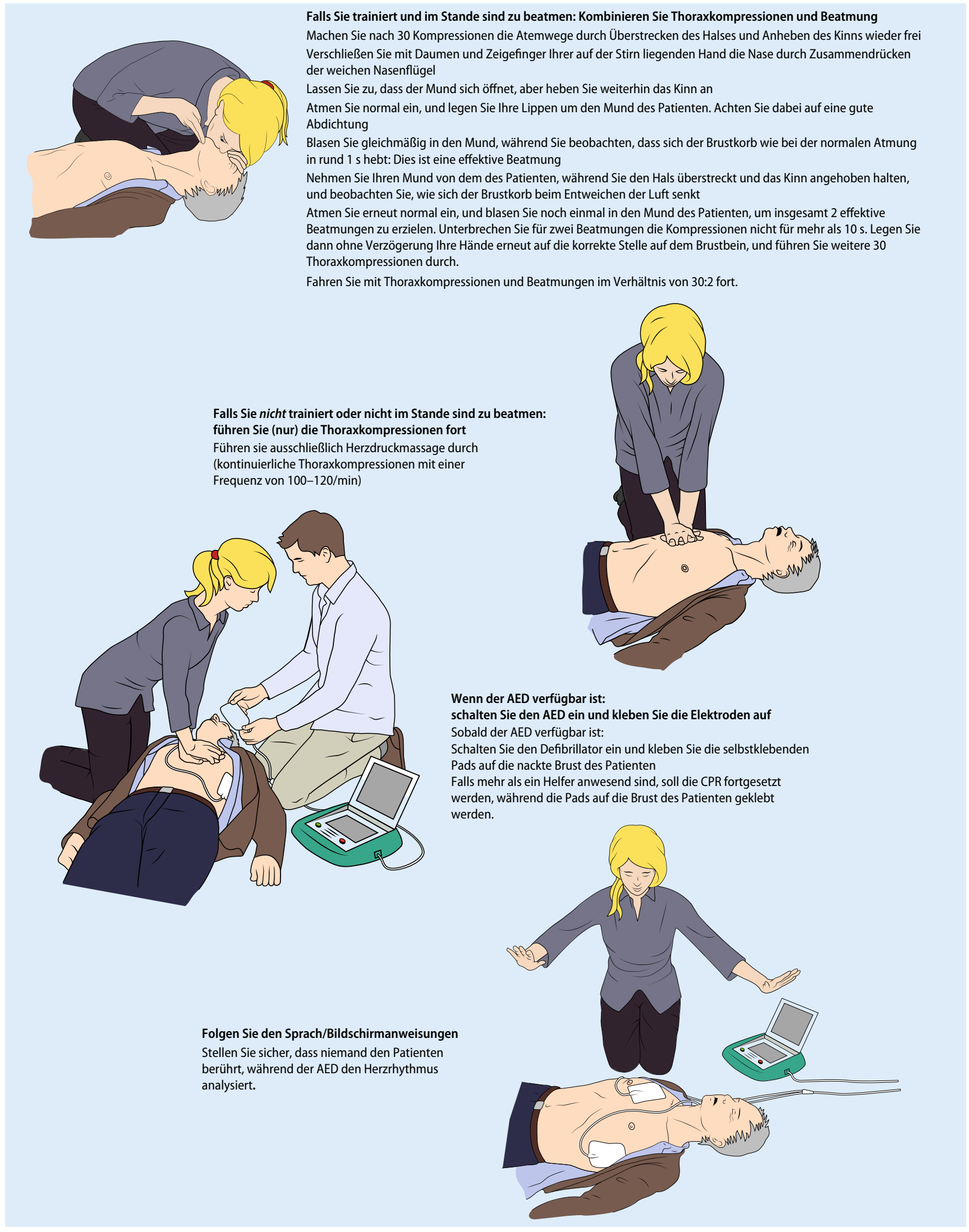

Abb. 4 \ (Fortsetzung Teil 2) 


\section{ERC Leitlinien}

Wird ein Schock empfohlen: Lösen Sie ihn aus Stellen Sie sicher, dass niemand den Patienten berührt. Drücken Sie den Auslöseknopf, wenn Sie dazu aufgefordert werden. (Vollautomatische AED geben den Schock automatisch ab.)

Starten Sie unverzüglich erneut CPR-30:2

Folgen Sie weiter den Sprach/Bildschirmanweisungen.

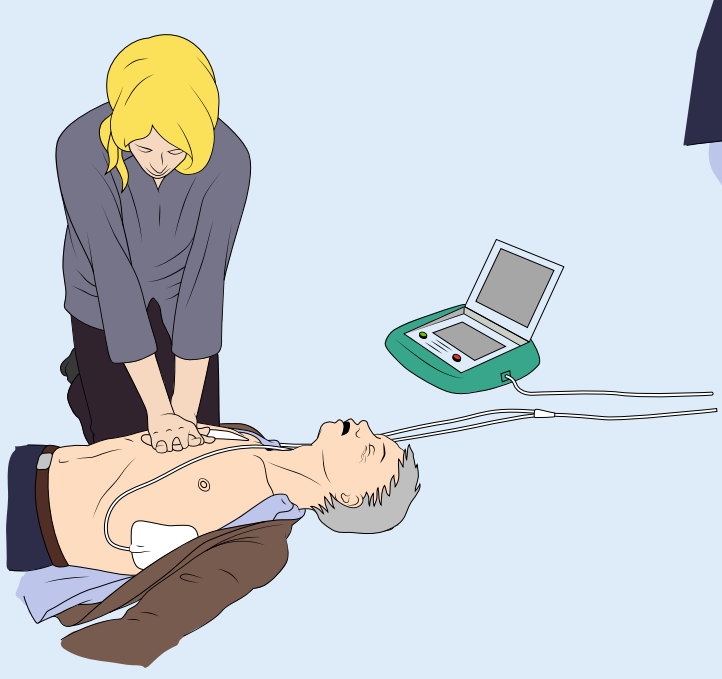

Ist kein AED verfügbar:

Fahren sie mit Thoraxkompressionen (und Beatmung) fort

Unterbrechen Sie die CPR-Maßnahmen nicht, bis:

- Ein professioneller Helfer Sie anweist, aufzuhören

- der Patient wirklich aufwacht: sich bewegt, die

Augen öffnet und normal zu atmen beginnt

- Sie erschöpft sind.

Wird kein Schock empfohlen: Führen Sie die CPR fort

Nehmen Sie unverzüglich die CPR wieder auf wie von den Sprach/Bildschirmanweisungen angegeben.

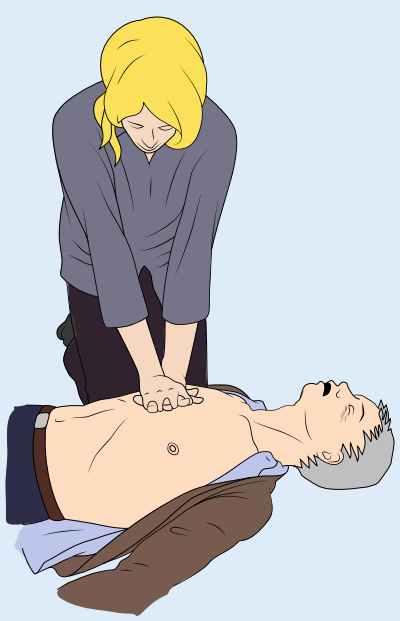

Wenn der Patient nicht reagiert aber normal atmet

Sind Sie sicher, dass der Patient normal atmet, aber nicht reagiert, drehen Sie ihn in die Seitenlage (Erste Hilfe Kapitel).

Es ist selten, das durch CPR allein wieder ein Kreislauf erreicht wird. Wenn Sie nicht wirklich sicher sind, fahren Sie mit CPR fort bis der Patient Zeichen der Erholung zeigt: - er wacht auf

- er bewegt sich

- er öffnet die Augen

- er atmet norma

Seien Sie bereit, sofort wieder mit CPR zu beginnen, wenn sich der Patient wieder verschlechtert.

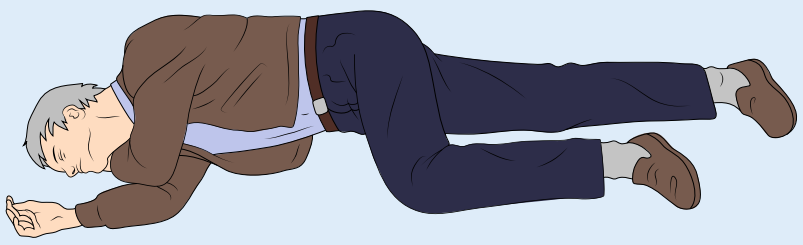

Abb. 4 A (Fortsetzung Teil 3) 
nicht mehr empfohlen. Prüfen Sie mit der Technik aus - Abb. 4 die Atmung. Seien Sie sich der kritischen Bedeutung bewusst, die oben beschriebene Schnappatmung zu erkennen.

\section{Alarmierung des Rettungsdienstes}

In der gesamten europäischen Union ist die kostenfreie Notrufnummer 112 eingerichtet. Aus dem Festnetz und über Mobiltelefone lassen sich die Notdienste - Rettungsdienst, Feuerwehr und Polizei - erreichen. Es gibt aber auch nationale Regelungen mit einer direkten Zugangsnummer für den Rettungsdienst, die möglicherweise Zeit spart. Notfallzeugen sollen deshalb den nationalen Leitlinien zur optimalen Telefonnummer folgen.

Früher Kontakt mit dem Rettungsdienst ermöglicht die Unterstützung durch den Leitstellendisponenten bei der Erkennung eines Kreislaufstillstands, der Telefonreanimation, der Alarmierung des Rettungsdienstes („First Responder") sowie beim Auffinden und Holen eines AED [75-78]. Wenn möglich, bleiben Sie beim Patienten, wenn Sie den Notruf absetzen. Schalten Sie den Lautsprecher des Telefons ein, um den Anweisungen des Disponenten stets folgen zu können, auch (wenn nötig) zur Thoraxkompression [79]. Es ist sinnvoll, bei Reanimationskursen das Einschalten des Lautsprechers zu üben [80]. Weitere Notfallzeugen können den Anruf des Rettungsdienstes übernehmen.

\section{Beginn der Thoraxkompression}

Wenn ein Erwachsener Herzdruckmassage benötigt, besteht in der Regel ein kardiales Problem. Wenn der Blutfluss beim Kreislaufstillstand stoppt, bleibt das Blut in der Lunge und in den Blutgefäßen noch für einige Minuten mit Sauerstoff gesättigt. Um den Vorrang der Herzdruckmassage bei der Wiederbelebung zu betonen, wird empfohlen, die Wiederbelebung mit Thoraxkompressionen statt mit Beatmung zu beginnen. Untersuchungen mit Übungsphantomen haben gezeigt, dass auf diese Weise auch schneller mit der Wiederbelebung begonnen wird [81-84].
Führen Sie Thoraxkompressionen folgendermaßen durch:

1. Drücken Sie auf die Mitte des Brustkobs.

2. Drücken Sie bei einem durchschnittlichen Erwachsenen ungefähr $5 \mathrm{~cm}$ tief, aber nicht tiefer als $6 \mathrm{~cm}$.

3. Komprimieren Sie den Thorax mit einer Frequenz von $100-120 / \mathrm{min}$, unterbrechen Sie so selten wie möglich.

4. Entlasten Sie nach jeder Kompression den Brustkorb vollständig; lehnen Sie sich nicht auf den Brustkorb.

\section{Handposition}

Experimentelle Studien haben gezeigt, dass die hämodynamischen Effekte besser sind, wenn der Druck auf die untere Hälfte des Brustbeins ausgeübt wird [85-87]. Es wird empfohlen, die Lokalisierung so einfach wie möglich zu lehren $[88,89]$, wie z. B.: „Legen Sie Ihre Handwurzel mitten auf den Brustkorb und die andere Hand darauf." Gleichzeitig demonstrieren Sie, wie Sie Ihre Hände auf der unteren Hälfte des Brustbeins platzieren [88, 89].

Ein einzelner Helfer kniet bei der Thoraxkompression am besten an der Seite des Patienten. So kann er am einfachsten und mit den geringsten Unterbrechungen zwischen Herzdruckmassage und Beatmung wechseln. Über-Kopf-Herzdruckmassage durch einen einzelnen Retter oder Herzdruckmassage mit gespreizten Beinen (Grätschschritt) durch zwei Helfer kann erwogen werden, wenn durch die räumliche Enge der seitliche Zugang nicht möglich ist $[90,91]$.

\section{Drucktiefe}

Die Angst, etwas falsch zu machen, Erschöpfung und mangelnde Muskelkraft führen häufig dazu, dass Helfer bei der Thoraxkompression nicht so tief drücken wie empfohlen. Nach den Leitlinien von 2010 zeigten vier Beobachtungsstudien, dass eine Drucktiefe von 4,5-5,5 cm bei Erwachsenen zu besseren Ergebnissen führte, als alle anderen angewandten Drucktiefen [92-95]. In einer Untersuchung an 9136 Patienten waren Drucktiefen von 4,0 bis $5,5 \mathrm{~cm}$ mit einem Maximum von 4,6 cm mit den höchsten Überlebensraten assoziiert [94]. Eine
Beobachtungsstudie beweist ferner, dass eine Drucktiefe von mehr als $6 \mathrm{~cm}$ bei der Thoraxkompression bei Erwachsenen zu mehr Schäden führt, als mit Drucktiefen von 5-6 cm [96]. Der ERC schließt sich der ILCOR-Empfehlung an, dass es bei einem durchschnittlich großen Erwachsenen sinnvoll ist, eine Drucktiefe von 5- bis maximal $6 \mathrm{~cm}$ anzustreben. Der ERC ist sich bei dieser Empfehlung bewusst, dass es schwierig sein kann, die Drucktiefe einzuschätzen, und dass zu geringe Drucktiefen eher schaden als zu tiefe. Im Training soll kontinuierlich die Bedeutung einer adäquaten Drucktiefe betont werden.

\section{Kompressionsfrequenz}

Die Kompressionsfrequenz ist definiert als die aktuelle Frequenz der Thoraxkompressionen, die in einem umschriebenen Zeitraum durchgeführt werden. Sie unterscheidet sich von der Zahl der Herzdruckmassagen in einem spezifischen Zeitraum, die auch die Unterbrechungen berücksichtigt.

Zwei Studien an insgesamt 13.469 Patienten fanden eine höhere Überlebensrate bei Patienten, die Thoraxkompressionen mit einer Frequenz von 100-120/min erhielten, verglichen mit $>140 / \mathrm{min}, 120-139 / \mathrm{min},<80 / \mathrm{min}$ oder $80-99 / \mathrm{min}$. Sehr hohe Kompressionsfrequenzen führten zu einer zu geringen Drucktiefe [97, 98]. Daher empfiehlt der ERC die Kompressionsfrequenz von 100-120/min.

\section{Unterbrechungen der Thoraxkompressionen minimieren} Atemspenden, Elektroschocks sowie Analysen der Beatmung und des Herzrhythmus führen $\mathrm{zu}$ Unterbrechungen der Herzdruckmassage. Pausen von weniger als $10 \mathrm{~s}$ vor und nach der Abgabe eines Schocks und ein Anteil der Thoraxkompressionen von mehr als $60 \%$ sind mit besserem Outcome verbunden [99-103]. Unterbrechungen der Thoraxkompressionen sollen minimiert werden, wobei erkannt werden muss, wie wichtig die Aufmerksamkeit und das Wechselspiel der Helfer ist, die zusammenarbeiten. 


\section{Fester Untergrund}

Wann immer möglich soll die Thoraxkompression auf einer harten Unterlage erfolgen. Luftgefüllte Matratzen müssen entlüftet werden [104]. Die Evidenz für den Nutzen von Rückenbrettern ist zweifelhaft [105-109]. Wenn sie verwendet werden, geben Sie acht, dass es nicht zu Unterbrechungen der Thoraxkompression und zur Dislokation von Atemwegsund Gefäßzugängen kommt.

\section{Brustkorbentlastung}

Während der Herzdruckmassage lehnt sich der Helfer häufig auf den Brustkorb, sodass sich dieser nicht wieder vollständig ausdehnen kann $[110,111]$. Kann sich die Brustwand nach jeder Kompression wieder komplett ausdehnen, führt dies jedoch zu einem besseren Rückfluss des Blutes zum Herzen, und das kann den Erfolg der Herzdruckmassage verbessern [110, 112-114]. Helfer sollen also darauf achten, sich nicht auf dem Brustkorb abzustützen.

\section{Kompressionsablauf}

Der optimale Kompressionsablauf (Verhältnis von Kompressionszeit zur Gesamtzeit von Kompression und Entlastung) wurde an Tiermodellen und mit mathematischen Simulationen mit uneinheitlichen Ergebnissen untersucht [115-123]. Eine Beobachtungsstudie an Menschen hat jüngst das bislang empfohlene Verhältnis von 50:50 infrage gestellt, sie kommt zu dem Schluss, dass Kompressionsphasen von mehr als $40 \%$ eines Zyklus nicht machbar seien und $\mathrm{zu}$ einer zu geringeren Drucktiefe führen könnten [124]. Für den Helfer ist das Verhältnis schwer zu korrigieren, es wird stark durch andere Parameter der Thoraxkompression beeinflusst. Der ERC räumt ein, dass es wenig Evidenz dafür gibt, ein spezifisches Verhältnis zu empfehlen, folglich genügt die Evidenz nicht, das bisher empfohlenen Verhältnisses von 50:50 zu zu ändern.

\section{Feedback der Kompressions- technik}

Die Verwendung von Geräten zu Feedback- und Sprachführung soll in der klinischen Praxis die Qualität der Wieder- belebung verbessern, indem die Chancen auf ROSC („return of spontaneous circulation“ - Rückkehr des Spontankreislaufs) und damit auf ein Überleben erhöht werden $[125,126]$. Die Art des Feedbacks schließt Sprachanweisungen, Metronome, Anzeigen, Displays, Wellendarstellungen, schriftliche Anweisungen und optische Alarme ein.

Der Effekt solcher Maßnahmen wurde in 2 randomisierten [92, 127] und 11 Beobachtungsstudien untersucht [128-138]. Keine dieser Studien zeigt mit Feedback ein verbessertes Überleben zum Zeitpunkt der Entlassung aus dem Krankenhaus, und nur eine Untersuchung fand eine signifikant höhere ROSC-Häufigkeit. In dieser Studie wurde es allerdings dem behandelnden Arzt überlassen, wann er ein solches Gerät einsetzt, und über die Gründe, wann ein Gerät eingesetzt wurde oder nicht, sagt die Studie nichts aus [136]. Der Einsatz eines Feedback-Geräts bei der Wiederbelebung sollte als Teil einer umfassenden Qualitätssteigerungsinitiative zur Wiederbelebung betrachtet werden $[138,139]$, nicht als isolierte Maßnahme.

\section{Atemspende}

Bei nicht relaxierten Schweinen mit Schnappatmung, mit ungesichertem, aber freiem Atemweg, führt kontinuierliche Thoraxkompression ohne Beatmung $\mathrm{zu}$ einem verbesserten Outcome [140]. $\mathrm{Zu}$ Beginn eines Kreislaufstillstands hat ein Drittel der Menschen eine Schnappatmung, die den Gasaustausch erleichtert [48]. Thoraxkompressionen führen aber beim intubierten Patienten nur zu einem Atemhubvolumen von $40 \mathrm{ml}$, nicht ausreichend für eine adäquate Ventilation [141]. Beim beobachteten Kreislaufstillstand im Kammerflimmern verdreifachte sofortige kontinuierliche Thoraxkompression das Überleben [142]. Dementsprechend mag sie besonders in der frühen (elektrischen) und zirkulatorischen Phase der Wiederbelebung vorteilhaft sein, während in der späteren (metabolischen) Phase eine zusätzliche Beatmung an Bedeutung gewinnt [39].

Während der Herzdruckmassage ist der systemische Blutfluss und damit der Blutzufluss zur Lunge deutlich re- duziert, folglich halten ein niedrigeres Atemhubvolumen und eine niedrigere Atemfrequenz als normal eine effektive Sauerstoffversorgung und Ventilation aufrecht [143-146]. Bei ungesicherten Atemwegen führt ein Atemhubvolumen von $1000 \mathrm{ml}$ häufiger zur Magenüberblähung als ein Hubvolumen von $500 \mathrm{ml}$ [147]. Eine Beatmungszeit von $1 \mathrm{~s}$ ist ohne übermäßige Magenblähung machbar [148]. Während einer Wiederbelebung kann es zu einer unbemerkten Hyperventilation kommen, besonders bei der Beutel-Masken-Beatmung eines Patienten mit gesichertem Atemweg (Intubation, supraglottische Atemwegshilfen). Obwohl dies zu einem erhöhten intrathorakalen Druck [149] und erhöhten Atemwegsdruckspitzen [150] führt, fanden sich in einer sorgfältig kontrollierten Tierstudie keine negativen Effekte [151].

Auf der Basis der vorhandenen Evidenz empfiehlt der ERC für die Beatmung eines Erwachsenen bei der Wiederbelebung ein Hubvolumen von 500 bis $600 \mathrm{ml}$ (6 bis $7 \mathrm{ml} / \mathrm{kg}$ ). In der Praxis führt dies zu einem sichtbaren Heben des Brustkorbs [152]. Notfallhelfer sollen eine Beatmungsdauer von $1 \mathrm{~s}$ anstreben, sodass sich der Brustkorb hebt, aber eine zu schnelle und zu heftige Beatmung vermieden wird. Für 2 Beatmungen sollen die Thoraxkompressionen nicht länger als $10 \mathrm{~s}$ unterbrochen werden [153]. Diese Empfehlung gilt für alle Formen der Beatmung während einer Wiederbelebung, einschließlich Mund-zu-Mundund Beutel-Masken-Beatmung mit oder ohne Sauerstoffgabe.

\section{Mund-zu-Nase-Beatmung}

Die Mund-zu-Nase-Beatmung stellt eine akzeptable Alternative zur Mund-zuMund-Beatmung dar [154]. Sie kann erwogen werden, wenn der Mund des Patienten schwer verletzt ist oder nicht geöffnet werden kann, der Patient sich im Wasser befindet oder bei der Mundzu-Mund-Beatmung keine Abdichtung erreicht werden kann.

\section{Mund-zu-Tracheostoma- Beatmung}

Ein Patient der eine Trachealkanüle oder ein Trachealstoma trägt, kann im 
Notfall Mund-zu-Tracheostoma beatmet werden [155].

\section{Kompressions-Beatmungs- Verhältnis}

Daten aus Tierversuchen sprechen dafür, dass das Verhältnis Thoraxkompression zu Ventilation größer als 15:2 sein sollte [156-158]. Nach einem mathematischen Modell bietet ein Verhältnis 30:2 den besten Kompromiss, was Blutfluss und Sauerstoffversorgung angeht $[159,160]$. In den Leitlinien 2005 und 2010 wurde dem auf sich allein gestellten Helfer ein Verhältnis 30:2 bei der Wiederbelebung eines Erwachsenen empfohlen. So werden Unterbrechungen der Thoraxkompression und damit die Zeiten ohne Blutfluss („no flow fraction“) [161, 162] sowie die Wahrscheinlichkeit einer Hyperventilation reduziert [149, 163]. Mehrere Beoabachtungsstudien berichten von einer leichten Verbesserung beim Outcome der Patienten, nachdem die Leitlinienänderung von 15:2 auf 30:2 eingeführt worden war [161, 162, 164, 165]. Der ERC bleibt daher bei seiner Empfehlung für ein Kompressions-Ventilations-Verhältnis von 30:2.

\section{Reanimation ohne Beatmung ("compression-only CPR ${ }^{\prime \prime}$ )}

Tierversuche zeigten, dass eine Wiederbelebung nur durch Thoraxkompression in den ersten wenigen Minuten nach einem nicht asphyktischen Kreislaufstillstand genauso effektiv sein kann wie eine Kombination aus Herzdruckmassagen und Beatmungen [140, 166]. Tierversuche und mathematische Modelle haben aber auch gezeigt, dass die Sauerstoffvorräte nach 2-4 min erschöpft sind [158, 167]. Bei freien Atemwegen und gelegentlicher Schnappatmung sowie durch passive Rückstellkräfte des Brustkorbs kann ein gewisser Gasaustausch stattfinden [48, 141, 168-170].

Beoabachtungsstudien, üblicherweise als sehr niedrige Evidenz eingestuft, deuten darauf hin, dass beim wahrscheinlich kardial bedingten Kreislaufstillstand eines Erwachsenen eine Wiederbelebung nur durch Herzdruckmassage und eine Wiederbelebung mit Herzdruckmassa- ge und Beatmung gleichwertig sind [26, 171-182].

Der ERC hat sorgfältig die möglichen Vor- und Nachteile der Wiederbelebung ohne Beatmung und der Standardwiederbelebung mit Beatmung abgewogen. Unser Vertrauen in die Gleichwertigkeit der Wiederbelebung mit und ohne Beatmung reicht nicht aus, um das bisher Empfohlene zu ändern. Der ERC unterstützt daher die ILCOR-Empfehlung, dass Helfer bei allen Patienten mit Kreislaufstillstand eine Herzdruckmassage durchführen sollen. Helfer, die trainiert und in der Lage sind zu beatmen, sollen Herzdruckmassage und Atemspenden durchführen, weil dies für Kinder und Patienten mit einem asphyktischen Kreislaufstillstand $[175,183,184]$ sowie bei spätem Eintreffen des Rettungsdienstes von Vorteil für den Patienten ist [179].

\section{Einsatz eines automatisierten externen Defibrillators}

AEDs sind sicher und effektiv, wenn sie durch Laien mit wenig oder ohne Training verwendet werden [185]. AEDs ermöglichen eine Defibrillation viele Minuten, bevor professionelle Hilfe eintrifft. Helfer sollen Thoraxkompressionen mit minimalen Unterbrechungen durchführen, während der AED angelegt und verwendet wird. Die Helfer sollen sich darauf konzentrieren, der Sprachführung unmittelbar $\mathrm{zu}$ folgen, insbesondere die Herzdruckmassage sofort wieder aufzunehmen, wenn dazu aufgefordert wird, und Unterbrechungen der Thoraxkompressionen zu minimieren. Pausen vor und nach einem Schock sollten so kurz wie nur möglich sein [99, 100, 103, 186]. Standard-AEDs können schon für Kinder ab 8 Jahren verwendet werden [187-189].

Für Kinder zwischen 1 und 8 Jahren sollen spezielle Klebeelektroden für Kinder verwendet werden, wenn möglich mit einem Kinderprogramm. Stehen sie nicht zur Verfügung, soll der Defibrillator verwendet werden, wie er ist. Die Verwendung des AED wird nicht für Kinder unter einem Jahr empfohlen, obwohl es Berichte über einen erfolgreichen Einsatz in dieser Altersgruppe gibt [190, 191]. Abgesehen von herzkran- ken Kleinkindern ist eine defibrillierbare Rhythmusstörung bei Kleinkindern extrem selten [187-189, 192-195]. Ist in diesen wenigen Fällen ein AED der einzige verfügbare Defibrillator, sollte sein Einsatz erwogen werden (vorzugsweise mit verringerter Dosis).

\section{Wiederbelebungsmaßnahmen vor der Defibrillation}

Die Bedeutung unmittelbarer Defibrillation wurde immer in Leitlinien und Ausbildung betont, ihr wird großer Einfluss auf das Überleben nach Kammerflimmern zugesprochen. 2005 wurde dieses Konzept infrage gestellt, da Evidenz dafür vorlag, dass Thoraxkompressionen von bis zu 180 s vor einer Defibrillation das Überleben verbessern können, wenn der Rettungsdienst erst nach mehr als 4-5 min eintrifft [196, 197]. Drei jüngere Studien konnten diesen Vorteil nicht bestätigen [198-200]. Die Analyse einer randomisierten Studie deutete auf eine Verschlechterung des Überlebens bis zur Krankenhausentlassung durch eine längere Periode CPR (Reanimation ohne Beatmung länger als $180 \mathrm{~s}$ ) mit dadurch verzögerter Defibrillation eines defibrillierbaren Rhythmus hin [200]. Allerdings war in Rettungsdienstbereichen mit einer hohen Ausgangsüberlebensrate bei Krankenhausentlassung (definiert als mehr als $20 \%$ der initial defibrillierbaren Fälle) eine Wiederbelebung über $180 \mathrm{~s}$ vor der Defibrillation erfolgreicher als eine Wiederbelebung über eine kürzere Zeit (30-60 s) [201]. Der ERC empfiehlt, dass CPR fortgeführt werden soll, während ein Defibrillator oder AED gebracht und angelegt wird, aber dann soll die Defibrillation nicht weiter verzögert werden.

\section{Intervall zwischen den Rhythmusanalysen}

Der ILCOR Consensus on Science 2015 berichtet, dass es derzeit keine Studien gibt, welche direkt zur Frage des optimalen Abstands von Rhythmusanalysen und deren Einfluss auf das Überleben Stellung nehmen: ROSC, gutes neurologisches und funktionelles Outcome, Überleben zum Zeitpunkt der Kranken- 
hausentlassung, Koronardurchblutung und Herzzeitvolumen.

Übereinstimmend mit der ILCOREmpfehlung und den bisherigen Leitlinien entsprechend empfiehlt der ERC, dass die Thoraxkompressionen alle $2 \mathrm{~min}$ für eine Rhythmusanalyse unterbrochen werden sollen.

\section{Sprachanweisungen}

Es ist von großer Wichtigkeit, dass die Notfallhelfer den Anweisungen des AED ohne Verzug folgen. Deshalb wird empfohlen, die Sprachführung so $\mathrm{zu}$ programmieren, dass sie die vorstehenden Zeitabläufe berücksichtigen. Dies schließt ein:

1. minimale Unterbrechung der Herzdruckmassagen zur Rhythmusanalyse und zum Laden,

2. 1 Schock, wenn ein defibrillierbarer Rhythmus erkannt wird,

3. die Anweisung zur sofortigen Wiederaufnahme der Thoraxkompressionen nach dem Schock,

4. 2 min Wiederbelebung bis zur nächsten Rhythmusanalyse.

Geräte, die die Qualität der Wiederbelebung messen, können zusätzlich Feedback in Echtzeit und weitere Sprach- oder Sichtanweisungen geben.

Einzelheiten zu Schocksequenzen, Energiestufen und der Dauer der Wiederbelebung zwischen den Schocks werden in Kap. 3, „Erweiterte Maßnahmen“, behandelt.

In der Praxis werden AEDs meist von ausgebildeten Helfern eingesetzt, sodass die AED-Sprachführung grundsätzlich auf ein Kompressions-Ventilations-Verhältnis von 30:2 eingestellt werden soll.

Wenn - ausnahmsweise - AEDs an einem Ort platziert werden, wo es unwahrscheinlich ist, dass ausgebildete Helfer dazukommen, kann der Betreiber die Einstellung auf Herzdruckmassage ohne Beatmung ändern lassen.

\section{Vollautomatische AEDs}

Hat ein vollautomatischer AED einen defibrillierbaren Rhythmus erkannt, gibt er den Schock ohne weiteres Zutun des Helfers ab. In einer Studie an Übungs- phantomen konnte gezeigt werden, dass ungeschulte Krankenpflegeschüler mit einem vollautomatischen AED weniger Fehler machten als mit einem halbautomatischen [202]. Eine simulierte Studie an Phantomen ergab, dass die Sicherheit nicht gefährdet war, wenn ungeübte Laienhelfer einen vollautomatischen statt einen halbautomatischen AED benutzten [203].

Über die Anwendung an Menschen in einem klinischen Bereich liegen keine Daten vor.

\section{Defibrillatoren in der Öffentlichkeit („public access defibrillation“, PAD)}

Die Bedingungen für eine erfolgreiche Wiederbelebung in einem Wohnumfeld sind schlechter als im öffentlichen Raum: weniger beobachtete Kreislaufstillstände, weniger Wiederbelebungen durch Notfallzeugen und als Konsequenz weniger defibrillierbare Kreislaufstillstände. Dies schränkt die Effektivität der AED-Anwendung im häuslichen Bereich ein [204]. Die meisten Untersuchungen, die einen positiven Effekt des AEDEinsatzes auf das Überleben zeigen, wurden mit AEDs in der Öffentlichkeit durchgeführt [32, 205-208]. Jüngere landesweite Untersuchungen aus Japan und den USA zeigen, dass, wenn ein AED verfügbar ist, die Patienten sehr viel früher defibrilliert werden und eine bessere Überlebenschance haben [16, 209]. Aber nur in 3,7 \% [209] bzw. 1,2\% [16] wurde ein Schock abgegeben. In der japanischen Studie findet sich eine eindeutig negative Beziehung zwischen der Anzahl der pro Quadratkilometer verfügbaren AEDs und dem Intervall zwischen dem Kollaps des Patienten und dem erstem Schock, was eine positive Korrelation mit dem Überleben ergab. Öffentliche AED-Programme sollen daher an viel besuchten Plätzen mit viel Publikumsverkehr eingerichtet werden (Flughäfen, Bahn- und Busstationen, Sportstätten, Einkaufszentren, Bürogebäuden und Kasinos). Dort werden Kreislaufstillstände häufig beobachtet, und trainierte Notfallhelfer sind schnell zur Stelle. Die Dichte, mit der AEDs für eine ausreichend schnelle Intervention zur Verfügung stehen müssen, ist be- sonders unter dem Aspekt der KostenNutzen-Relation nicht zufriedenstellend definiert. Die Häufigkeit, mit der ein Kreislaufstillstand erwartet wird, welchen Gewinn an Lebensjahren man erhofft und welchen Zeitgewinn ein mit einem AED ausgerüsteter Notfallhelfer gegenüber dem Regelrettungsdienst hat, sollen in die Entscheidung einfließen. Die Verfügbarkeit eines AED an Orten, an denen sich ein Kreislaufstillstand alle 5 Jahre ereignet, kann als kosteneffektiv und anderen medizinischen Interventionen vergleichbar erachtet werden [210-221]. Für den häuslichen Bereich können Erfahrungen aus der Vergangenheit und die Art der Nachbarschaft bei der Entscheidung, wo ein AED stationiert sein soll, helfen [213, 214]. Die Registrierung der AEDStandorte erleichtert es dem Leitstellendisponenten, einen Notfallhelfer zum nächstplatzierten $\mathrm{AED} z u$ führen und somit die Hilfeleistung zu beschleunigen [215]. Die frühzeitige Defibrillation mit einem AED vor Ort kann möglicherweise auch Krankenhauskosten reduzieren [216, 217].

Das volle Potenzial von AEDs ist noch nicht ausgeschöpft, da sie meist im öffentlichen Raum zum Einsatz kommen sich aber 60 bis $80 \%$ der Kreislaufstillstände zu Hause ereignen. Der Anteil der Patienten, die mit Kammerflimmern aufgefunden werden, ist zu Hause geringer als in der Öffentlichkeit, wohingegen die absolute Zahl zu behandelnder Patienten zu Hause höher ist [204]. Selten profitieren Patienten zu Hause von öffentlichen AED-Programmen [208]. Daher sind andere Strategien gefragt, um eine frühzeitige Defibrillation in häuslicher Umgebung zu erreichen. Von der Leitstelle eingesetzte sog. „First Responder“ (Polizei, Feuerwehr) haben in der Regel längere Reaktionszeiten, erreichen aber die gesamte Bevölkerung [17, 36]. Die logistische Herausforderung für First-Responder-Programme liegt darin, dass der Notfallhelfer nicht unbedingt schneller als der Rettungsdienst eintreffen muss, aber bis 5-6 min nach dem Notruf, um den Defibrillationsversuch in der elektrischen bzw. zirkulatorischen Phase des Kreislaufstillstands zu ermöglichen [39]. Späteres Eintreffen führt zu verschlech- 
terten Überlebenschancen: Wenige Minuten Zeitgewinn haben wenig Bedeutung, wenn der First Responder später als 10 min nach dem Notruf eintrifft [34, 218]. Von der Leitstelle geführte Laienhelfer aus der Umgebung des Patienten, die zu einem nahe gelegenen AED geführt werden, können die Zahl der Wiederbelebung durch Laienhelfer verbessern und die Zeit bis zur Defibrillation verkürzen [40].

Wenn ein AED-Programm eingeführt wird, sollen Gemeinde und Verantwortliche berücksichtigen, dass ein Team für die Wartung der Geräte, für die Ausund Fortbildung von Personen, welche für den Einsatz des AED infrage kommen, und für die Bildung einer Gruppe Freiwilliger, die sich verpflichten, einen AED bei der Wiederbelebung von Patienten mit Kreislaufstillstand anzuwenden, notwendig ist [219]. Es müssen Rücklagen gebildet werden, die einen dauerhaften Unterhalt solcher Programme finanziell absichern.

Programme, die AEDs in häuslicher Umgebung bereitstellen, wurden lediglich im Hinblick auf die Reaktionszeiten, aber nicht auf Überlebensvorteile hin untersucht [40]. Die Bereitstellung eines AED für Einzelne zu Hause ist auch bei jenen, die ein hohes Risiko haben, einen plötzlichen Kreislaufstillstand $\mathrm{zu}$ erleiden, nicht effektiv [220].

Das Kap. „Besondere Umstände“ liefert die Evidenz, die die ERC-Empfehlung untermauert, dass an Bord aller kommerziellen Flugzeuge in Europa bindend AEDs vorgehalten werden sollen, auch bei Regionallinien und sog. „Billigfliegern" [221].

\section{Einheitliche AED-Kennzeichnung}

Wenn ein Patient kollabiert, muss schnell ein AED verfügbar sein: ein klares, einfaches Symbol muss auf seinen Standort und den schnellsten Weg dorthin hinweisen. ILCOR hat ein solches AED-Symbol entwickelt, das weltweit verstanden wird; daher wird dieses empfohlen [222].

\section{Einsatz von AEDs in Kranken- häusern}

Randomisierte Studien zum Einsatz von AEDs in Krankenhäusern im Vergleich zu manuellen Defibrillatoren gibt es nicht. Zwei ältere Beobachtungsstudien bei Erwachsenen mit Kreislaufstillstand im Krankenhaus und einer defibrillierbaren Rhythmusstörung zeigten eine höhere Überlebensrate bis zur Krankenhausentlassung, wenn es statt alleiniger manueller Defibrillation auch ein AEDProgramm gab [223, 224]. Eine jüngere Beobachtungsstudie konnte zeigen, dass ein AED erfolgreich eingesetzt werden konnte, bevor das innerklinische Wiederbelebungsteam eintraf [225]. Drei Beobachtungsstudien zeigten beim Vergleich von AED-Einsatz mit manueller Defibrillation keine Verbesserung beim Überleben bis zur Krankenhausentlassung [226-228]. In einer dieser Studien [226] überlebten in der AED-Gruppe weniger Patienten mit nicht defibrillierbarem Rhythmus bis zur Krankenhausentlassung als in der Gruppe mit manueller Defibrillation (15 vs. $23 \%$; $p=0,04)$. Eine andere große Beobachtungsstudie mit 11.695 Patienten in 204 Krankenhäusern zeigte auch, dass der AED im Krankenhaus mit einer niedrigeren Überlebensrate assoziiert war als ohne AED (16,3 vs.19,3\%; adjusted rate ratio, RR, 0,85; $95 \%$-Konfidenzintervall, CI, 0,78-0,92; $p<0,001$ [229]). Lag ein nicht defibrillierbarer Rhythmus vor, führte der AED-Einsatz zu einer geringeren Überlebensrate (10,4 vs.15,4\%; adjusted RR, 0,74; $95 \%-C I, 0,65-0,83$; $p<0,001)$ und $\mathrm{zu}$ einer vergleichbaren Überlebensrate bei defibrillierbarem Rhythmus (38,4 vs. 39,8\%; adjusted RR, 1,00; $95 \%$-CI, 0,88-01,13; $p=0,99$ ). Dies legt nahe, dass der AED eine nachteilige Verzögerung beim Beginn der Wiederbelebungsmaßnahmen (CPR) verursacht oder zu Unterbrechungen der Thoraxkompression bei nicht defibrillierbaren Rhythmen führt [230]. Weniger als $20 \%$ der Krankenhauspatienten, die einen Kreislaufstillstand erleiden, haben einen defibrillierbaren Rhythmus [229, 231, 232]. Wir empfehlen den Einsatz eines AED in den Bereichen eines Krankenhauses, in denen das Risiko einer verzögerten Defibrillation besteht [233], weil es mehrere Minuten dauert, bis ein Wiederbelebungsteam eintrifft, und Ersthelfer nicht in der Lage sind, manuell $\mathrm{zu}$ defibrillieren. Ziel ist eine
Defibrillation innerhalb von drei Minuten nach dem Kollaps. In Bereichen, in denen die manuelle Defibrillation durch trainiertes Personal oder Wiederbelebungsteams rasch durchgeführt werden kann, ist die manuelle Defibrillation dem AED-Einsatz vorzuziehen. Egal, welche Defibrillationstechnik bevorzugt wird (und einige Krankenhäuser mögen Defibrillatoren wählen, die AED und manuelle Defibrillation bieten), es muss ein effektives Aus- und Fortbildungsprogramm etabliert werden [232, 234]. Eine ausreichende Anzahl an Mitarbeitern muss ausgebildet werden, damit an jeder Stelle des Krankenhauses in $3 \mathrm{~min}$ eine Defibrillation durchgeführt werden kann. Krankenhäuser sollen die Zeiten von einem Kollaps bis zum ersten Schock registrieren und die Ergebnisse von Wiederbelebungsmaßnahmen überwachen und aufarbeiten.

\section{Risiken für den Ersthelfer und den Reanimationspatienten}

\section{Risiken für den Patienten, der wiederbelebt wird, obwohl er keinen Kreislaufstillstand hat}

Viele Notfallzeugen beginnen nicht mit der Wiederbelebung, weil sie befürchten, dass Thoraxkompressionen bei einem Patienten, der keinen Kreislaufstillstand hat, ernste Schäden verursachen. Drei Untersuchungen befassten sich mit den Risiken einer Herzdruckmassage bei Personen, die keinen Kreislaufstillstand hatten [235-237]. In den gepoolten Daten dieser drei Studien, also von 345 Patienten, fanden sich Knochenbrüche (Rippen und Schlüsselbein) mit 1,7 \% (95\%CI, 0,4-3,1\%), Schmerzen an der Stelle der Herzdruckmasage mit 8,7\% (95\%CI, 5,7-11,7\%), aber keine relevanten Verletzungen innerer Organe. Ersthelfer sollten keine Bedenken haben, mit einer Wiederbelebung zu beginnen, da es nur in extrem seltenen Fällen zu ernsthaften Verletzungen kommt, wenn ein Patient keinen Kreislaufstillstand hat und von einem Notfallzeugen wiederbelebt wird. 
Risiken für einen Patienten, der bei Vorliegen eines Kreislaufstillstands wiederbelebt wird

Eine systematische Übersicht zu Skelettverletzungen durch manuelle Thoraxkompression berichtet von 13-97\% Rippenbrüchen und von 1-43\% Sternumfrakturen [238]. Organverletzungen (Lunge, Herz, Bauchorgane) sind sehr viel seltener und kommen mit und ohne Knochenverletzungen vor [239]. Sie treten häufiger auf, wenn beim normalen Erwachsenen tiefer als $6 \mathrm{~cm}$ gedrückt wird [96].

\section{Risiken für den Helfer während des Trainings und bei realer Reanimation}

Beobachtungsstudien zur Ausbildung und tatsächlichen Durchführung von Wiederbelebungsmaßnahmen sowie Fallberichte dokumentieren nur selten Muskelzerrungen, Rückenbeschwerden, Kurzatmigkeit, Pneumothorax, Brustschmerzen, Herzinfarkt oder Nervenschäden [240, 241]. Die Häufigkeit solcher Ereignisse ist niedrig, und die Ausbildung in Wiederbelebungsmaßnahmen und deren tatsächliche Durchführung ist unter den meisten Umständen sicher [242]. Teilnehmer von Wiederbelebungsschulungen sollten über Art und Ausmaß der körperlichen Belastung während des Trainingsprogramms aufgeklärt werden. Lernenden und Helfern, die während des Trainings signifikante Symptome entwickeln (z. B. Brustschmerz oder starke Atemnot), soll zum Trainingsabbruch geraten werden.

\section{Ermüdung des Helfers}

Mehrere Studien am Übungsphantom haben nachgewiesen, dass die Drucktiefe bereits weniger als 2 min nach Beginn der Thoraxkompressionen abnimmt [243]. Eine Krankenhauspatientenstudie zeigte, dass auch bei Echtzeit-Feedbacks die durchschnittliche Tiefe der Herzdruckmassage zwischen 1,5 und 3 min nach Beginn der CPR nachließ [244]. Es wird daher empfohlen, dass sich Ersthelfer etwa alle 2 min abwechseln, um eine Verschlechterung der Druckqualität infolge der Ermüdung des Helfers zu verhindern. Beim Wechsel der Helfer soll die
Herzdruckmassage nicht unterbrochen werden.

\section{Risiken während der Defibrillation}

Viele Studien zu öffentlich zugänglichen Defibrillatoren („public access defibrillation", PAD) zeigen, dass AEDs von Laien und professionellen Ersthelfern (First Respondern) sicher angewendet werden können [185]. Eine systematische Metaanalyse fand 8 Publikationen, die insgesamt 29 unerwünschte Ereignisse bei der Defibrillation auswiesen [245]. Ursache waren zufälliger oder vorsätzlicher Missbrauch des Defibrillators, Gerätefehlfunktion und versehentliche Entladung während des Trainings oder der Wartung. In 4 Einzelfallberichten kam es durch die Entladung implantierter Herzschrittmacher (implantierbarer Kardioverter-Defibrillator, ICD) zu Schocks an Helfern, was in einem Fall zu einer Schädigung peripherer Nerven führte. Es gibt keine Berichte über Schädigungen der Ersthelfer durch Defibrillationsversuche in feuchter Umgebung.

Obgleich Verletzungen der Helfer durch Defibrillationen extrem selten sind, konnte gezeigt werden, dass chirurgische Handschuhe keinen ausreichenden Schutz bieten [246-249]. Daher sollen Helfer während der Schockabgabe die Thoraxkompressionen nicht fortsetzen, und der Patient soll während der ICD-Entladung nicht berührt werden. Direkter Kontakt zwischen dem Helfer und dem Patienten während der Schockabgabe soll vermieden werden.

\section{Psychologische Folgen}

Eine große prospektive Studie zu PAD berichtete von wenigen negativen psychologischen Effekten in Verbindung mit einer Wiederbelebung oder dem Einsatz eines AED, die eine Intervention erforderten [242]. Zwei große retrospektive Fragebogenstudien zur Wiederbelebung stellten fest, dass Notfallzeugen ihre Wiederbelebung als positive Erfahrung einstuften [250,251]. Auch Familienangehörige, die Zeugen von Wiederbelebungsmaßnahmen werden, können psychologisch davon profitieren [252-254]. Das seltene Auftreten von nachteiligen psychologischen Auswirkungen bei Ersthel- fern nach einer Wiederbelebung soll dennoch registriert und angemessen behandelt werden.

\section{Übertragung von Krankheiten}

Das Risiko einer Krankheitsübertragung während des Trainings und der tatsächlichen Wiederbelebung ist extrem niedrig [255-257]. Das Tragen von Handschuhen während der Wiederbelebung ist sinnvoll, aber die Wiederbelebung soll nicht verzögert oder gar unterlassen werden, weil keine Handschuhe verfügbar sind.

\section{Infektionsbarrieren für die Atemspende}

Drei Studien zeigten unter kontrollierten Laborbedingungen, dass Beatmungsfolien oder -ventile die Übertragung von Bakterien verringern $[258,259]$. Es konnten keine Studien gefunden werden, die die Sicherheit und Effektivität solcher Hilfsmittel (Beatmungstücher, Taschenmasken), die den direkten Kontakt mit dem Patienten verhindern sollen, untersuchten. Wenn man weiß, dass der Patient eine schwerwiegende Infektion hat (z. B. HIV, Tuberkulose, Hepatitis-BVirus, SARS), wird ein Infektionsschutz empfohlen.

Wird eine solche Infektionsbarriere verwendet, ist dafür Sorge zu tragen, dass es dadurch nicht zu unnötigen Unterbrechungen der Wiederbelebung kommt. Studien an Übungsphantomen haben gezeigt, dass die Qualität einer Wiederbelebung besser ist, wenn eine Taschenmaske verwendet wird, als wenn Beutel-Masken-Beatmung oder ein Gesichtstuch zur Anwendung kommt [260-262].

\section{Atemwegsverlegung durch Fremdkörper (Ersticken)}

Die Verlegung der Atemwege durch einen Fremdkörper ist eine seltene, aber potenziell behandelbare Todesursache [263]. $\mathrm{Da}$ die meisten Atemwegsverlegungen beim Essen entstehen, werden sie üblicherweise beobachtet. Da die Betroffenen anfangs bei Bewusstsein sind und reagieren, besteht oft die Möglichkeit zur frühzeitigen Intervention, die lebensrettend sein kann. 


\section{Verdacht auf Ersticken}
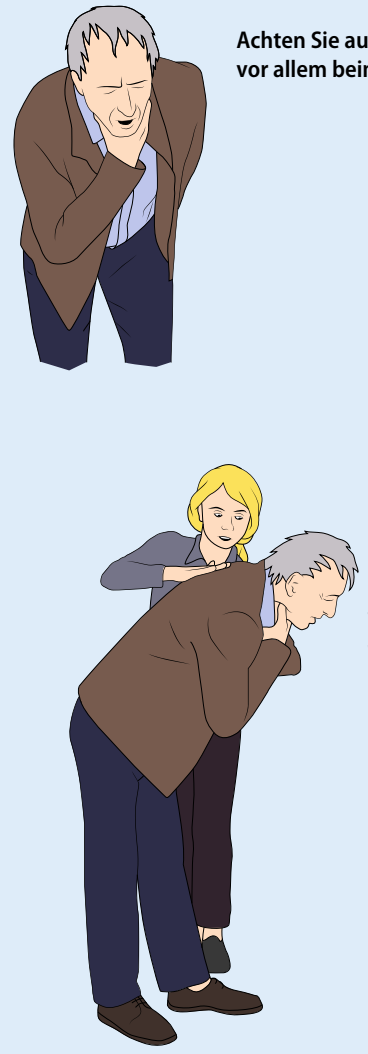

Bestärken Sie den Patienten, zu husten
Achten Sie auf Erstickungszeichen, vor allem beim Essen

Heimlich Handgriff Sind die Rückenschläge wirkungslos, geben Sie 5 Oberbauchstöße Falls die Atemwegsverlegung mit 5 Schlägen auf den Rücken nicht beseitigt werden kann, führen Sie bis zu 5 Kompressionen des Oberbauchs durch:

Stellen Sie sich hinter den Patienten und legen Sie beide Arme um seinen Oberbauch;

Lehnen Sie den Patienten nach vorn;

Ballen Sie die Faust und legen Sie sie zwischen Nabel und Brustkorb; Greifen Sie diese Hand mit Ihrer anderen und ziehen Sie kräftig nach innen und oben:

Wiederholen Sie dies bis zu 5-mal.

Falls die Verlegung immer noch nicht beseitigt ist, fahren Sie abwechselnd mit 5 Rückenschläge und 5 Oberbauchkompressionen fort.
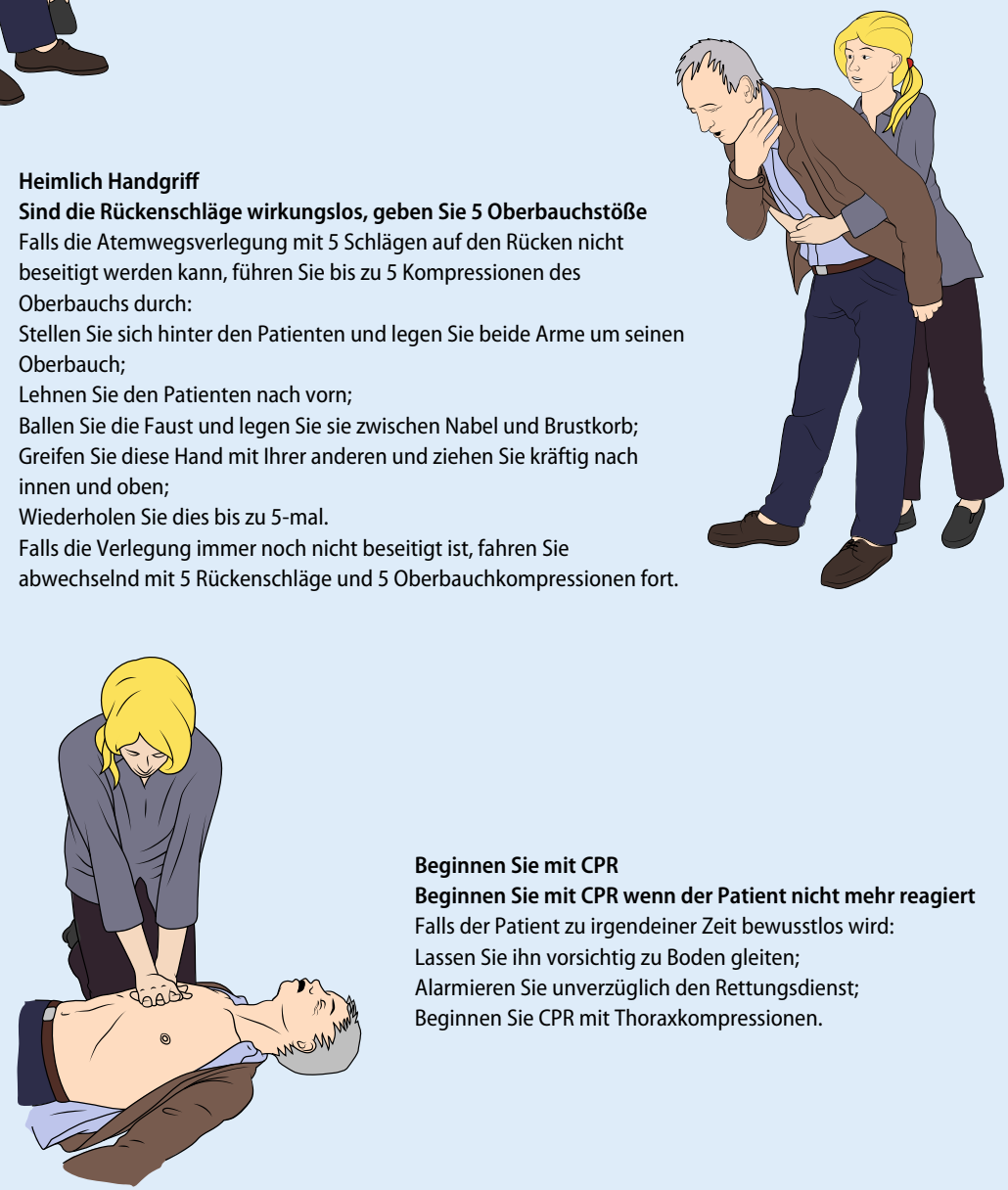

Beginnen Sie mit CPR

Beginnen Sie mit CPR wenn der Patient nicht mehr reagiert

Falls der Patient zu irgendeiner Zeit bewusstlos wird:

Lassen Sie inn vorsichtig zu Boden gleiten;

Alarmieren Sie unverzüglich den Rettungsdienst;

Beginnen Sie CPR mit Thoraxkompressionen.
Abb. $5<$ Schritt-fürSchritt-Erklärung des Behandlungsablaufs beim Erwachsenen mit Atemwegsverlegung durch Fremdkörper a Erkennen der Atemwegsverlegung. b Bei leichter Atemwegsverlegung ermutigen Sie den Patienten zu husten. c Bei schwerer Atemwegsverlegung schlagen Sie auf den Rücken. d Kombinieren sie die Rückenschläge mit Oberbauchkompressionen 


\section{Erkennen}

Der Schlüssel zum erfolgreichen Outcome liegt im Erkennen einer Atemwegsverlegung. Deshalb ist es wichtig, diesen Notfall nicht mit einer Ohnmacht, einem Herzinfarkt, einem Krampfanfall oder anderen Zuständen zu verwechseln, die plötzliche Atemnot, Zyanose oder Bewusstseinsverlust hervorrufen können. Die Fremdkörperverlegung ereignet sich üblicherweise beim Essen oder Trinken. Ein erhöhtes Risiko für eine Atemwegsverlegung durch Fremdkörper haben Patienten mit eingeschränktem Bewusstsein, Intoxikationen (Alkohol, Drogen), neurologischen Erkrankungen mit Störungen der Schluck- und Hustenreflexe (Schlaganfall, Parkinson-Krankheit), Atemwegserkrankungen, geistigen Einschränkungen, Demenz, schlechtem Zahnstatus und hohem Alter [264].

- Abb. 5 zeigt den Behandlungsalgorithmus bei einem Erwachsenen mit Atemwegsverlegung durch Fremdkörper. Fremdkörper können eine milde oder eine schwere Atemwegsverlegung verursachen. Es ist wichtig, den ansprechbaren Patienten zu fragen: „Haben Sie einen Erstickungsanfall?" Ein Patient, der antwortet, hustet und atmet, hat eine milde Obstruktion. Kann er nicht sprechen, nur schwach husten, ringt er nach Luft oder kann nicht atmen, so liegt eine schwere Obstruktion vor.

\section{Behandlung der milden \\ Atemwegsverlegung}

Husten erzeugt einen hohen und anhaltenden Atemwegsdruck und kann den Fremdkörper ausstoßen. Eine aggressive Behandlung mit Schlägen auf den Rücken, Oberbauch- und Brustkorbkompressionen kann Schäden hervorrufen und die Atemwegsverlegung verschlimmern. Diese soll Patienten vorbehalten bleiben, die Zeichen einer schweren Atemwegsverlegung aufweisen. Patienten mit einer milden Verlegung des Atemwegs sollen unter kontinuierlicher Beobachtung bleiben, bis es ihnen besser geht, weil sich eine schwere Verlegung noch entwickeln kann.
Behandlung der schweren

Atemwegsverlegung

Klinische Daten zum Ersticken sind größtenteils retrospektiv und anekdotisch. Bei Erwachsenen und Kindern über 1 Jahr, die bei Bewusstsein sind und bei denen eine komplette Atemwegsverlegung durch Fremdkörper erfolgt ist, haben Fallberichte die Effektivität von Schlägen auf den Rücken sowie Oberbauch- und Brustkorbkompressionen gezeigt [265]. In ungefähr 50 \% der Fälle kann die Atemwegsverlegung nicht durch eine einzige Maßnahme beseitigt werden [266]. Die Erfolgsaussichten steigen bei der Kombination von Schlägen auf den Rücken, Oberbauch- und Brustkorbkompressionen [265].

\section{Behandlung der Atemwegs- verlegung beim Bewusstlosen}

Eine randomisierte Studie an Leichen [267] und zwei prospektive Studien an anästhesierten Freiwilligen [268, 269] haben gezeigt, dass mit Brustkorbkompressionen im Vergleich zu Oberbauchkompressionen ein höherer Atemwegsdruck erzeugt werden kann. Herzdruckmassagen bei bewusstlosen oder nicht ansprechbaren Patienten mit einer Atemwegsverlegung durch Fremdkörper sind mit einem guten neurologischen Outcome assoziiert (Odds Ratio, 10,57; $95 \%$-CI, 2472-65.059, $p<0,0001$ ) [270]. Daher soll sofort mit Thoraxkompressionen begonnen werden, wenn der Patient nicht mehr reagiert oder bewusstlos wird. Nach 30 Kompressionen versuchen Sie zweimal zu beatmen. Führen Sie die Reanimation fort, bis sich der Patient erholt und normal zu atmen beginnt.

\section{Nachsorge und Vorstellung beim Arzt}

Nach erfolgreicher Beseitigung einer Atemwegsverlegung durch Fremdkörper können immer noch Fremdkörper in den oberen oder unteren Atemwegen verblieben sein und später zu Komplikationen führen. Patienten mit anhaltendem Husten, Schluckbeschwerden oder dem Gefühl, dass immer noch etwas im Hals steckt, sollen daher einem Arzt vorgestellt werden. Oberbauchkompressionen und Herzdruckmassagen können $\mathrm{zu}$ ernsthaften inneren Verletzungen führen; daher sollen alle Patienten, bei denen diese angewendet wurden, anschließend auf Verletzungen untersucht werden.

\section{Wiederbelebung von \\ Kindern (s. Kap. 6) und \\ Ertrinkungsopfern (s. Kap. 4)}

Viele Kinder werden nicht reanimiert, weil potenzielle Helfer fürchten, Schaden anzurichten, da sie nicht speziell in der Wiederbelebung von Kindern geschult sind. Diese Furcht ist unbegründet: Es ist viel besser, ein Kind nach dem BLS-Schema für Erwachsene zu reanimieren, als nichts zu tun. Um das Lernen und Erinnern zu vereinfachen, soll Laien beigebracht werden, dass die Erwachsenenmethode auch bei nicht reagierenden und nicht normal atmenden Kindern angewandt werden kann. Folgende geringe Änderungen an der Erwachsenensequenz machen diese für Kinder noch geeigneter:

- Beatmen Sie 5-mal, bevor sie mit den Thoraxkompressionen beginnen.

- Reanimieren Sie 1 min lang, bevor

Sie Hilfe holen, falls Sie wirklich ganz allein sind.

- Komprimieren Sie den Brustkorb, um ein Drittel; benutzen Sie 2 Finger bei Kleinkindern unter einem Jahr. Bei älteren Kindern sind 1 oder 2 Hände erforderlich, je nachdem, wie Sie eine ausreichende Kompressionstiefe erreichen.

Die gleichen Modifikationen - also 5 initiale Beatmungen sowie 1-minütige Wiederbelebungsmaßnahmen, bevor Sie Hilfe holen, falls Sie wirklich ganz allein sind - können das Outcome von Patienten nach Ertrinkungsunfällen verbessern. Diese Modifikation soll nur Helfern vermittelt werden, die eine spezielle Verpflichtung haben, sich um potenzielle Ertrinkungsopfer zu kümmern (z. B. Rettungsschwimmer). 


\section{Korrespondierender Übersetzer}

Dr. med. Ulrich Jost

DLRG

Im Niedernfeld 1-3

Bad Nenndorf

ulrich.jost@dlrg.de

\section{Korrespondenzadresse}

\section{G. Perkins}

Warwick Medical School, University of Warwick Coventry, UK

ulrich.jost@dlrg.de

\section{Einhaltung ethischer Richtlinien}

Interessenkonflikt. G.D. Perkins ist Editor der Resuscitation; A. J. Handley ist medizinischer Berater bei BA, Virgin, Places for people, Life saving Societies, Trading Company Secretary RCUK; G. Ristagno berät ZOLL: ECG interpretation; J. Soar ist Editor der Resuscitation; M. Castren ist im Medical advisory Board der Falck Foundation; R. Koster ist medizinischer Berater bei Physio Control and HeartSine und erhält Forschungsgelder von Physio Control, Philips, Zoll, Cardiac Science, Defibtech, Jolife; V. Wenzel erhält Forschungsgelder, ist medizinischer Berater und Vortragender bei „AOP Orphan“ Pharma; J.-T. Grasner, K.Monsieurs, M. Smyth, T. M.Olasveengen und V. Raffay geben an, dass kein Interessenkonflikt besteht.

Dieser Beitrag beinhaltet keine Studien an Menschen oder Tieren.

\section{Literatur}

1. Soar J, Nolan JP, Bottiger BW et al (2015) European Resuscitation Council Guidelines for Resuscitation 2015 Section 3 Adult Advanced Life Support. Resuscitation 95:99-146

2. Zideman DA, De Buck EDJ, Singletary EM et al (2015) European Resuscitation Council Guidelines for Resuscitation 2015 Section 9 First Aid. Resuscitation 95:277-286

3. Perkins GD, Travers AH, Considine J et al (2015) Part 3: adult basic life support and automated external defibrillation: 2015 International Consensus on Cardiopulmonary Resuscitation and Emergency Cardiovascular Care Science With Treatment Recommendations. Resuscitation 95:e43-e70

4. Berdowski J, Berg RA, Tijssen JG, Koster RW (2010) Global incidences of out-of-hospital cardiac arrest and survival rates: systematic review of 67 prospective studies. Resuscitation 81:1479-1487

5. Grasner JT, Herlitz J, Koster RW, Rosell-Ortiz F, Stamatakis L, Bossaert L (2011) Quality managementin resuscitation-towardsa European cardiac arrest registry (EuReCa). Resuscitation 82:989-994

6. Grasner JT, Bossaert L (2013) Epidemiology and management of cardiac arrest: what registries are revealing. Best Pract Res Clin Anaesthesiol 27:293-306

7. Cobb LA, Fahrenbruch CE, Olsufka M, Copass MK (2002) Changing incidence of out-of-hos- pital ventricular fibrillation, 1980-2000. JAMA 288:3008-3013

8. Rea TD, Pearce RM, Raghunathan TE et al (2004) Incidence of out-of-hospital cardiac arrest. Am J Cardiol 93:1455-1460

9. Vaillancourt C, Verma A, Trickett J et al (2007) Evaluating the effectiveness of dispatch-assisted cardiopulmonary resuscitation instructions. Acad Emerg Med 14:877-883

10. Agarwal DA, Hess EP, Atkinson EJ, White RD (2009) Ventricular fibrillation in Rochester, Minnesota: experience over 18 years. Resuscitation 80:1253-1258

11. Ringh $M$, Herlitz J, Hollenberg J, Rosenqvist M, Svensson L (2009) Out of hospital cardiac arrest outside home in Sweden, change in characteristics, outcome and availability for public access defibrillation. Scand J Trauma Resusc Emerg Med 17:18

12. Hulleman M, Berdowski J, de Groot JR et al (2012) Implantable cardioverter-defibrillators have reduced the incidence of resuscitation for out-of-hospital cardiac arrest caused by lethal arrhythmias. Circulation 126:815-821

13. Blom MT, Beesems SG, Homma PC et al (2014) Improved survival after out-of-hospital cardiac arrest and use of automated external defibrillators. Circulation 130:1868-1875

14. Cummins R, Thies W (1991) Automated externa defibrillators and the Advanced Cardiac Life Support Program: a new initiative from the American Heart Association. Am J Emerg Med 9:91-93

15. Waalewijn RA, Nijpels MA, Tijssen JG, Koster RW (2002) Prevention of deterioration of ventricular fibrillation by basic life support during out-ofhospital cardiac arrest. Resuscitation 54:31-36

16. Weisfeldt ML, Sitlani CM, Ornato JP et al (2010) Survival after application of automatic external defibrillators before arrival of the emergency medical system: evaluation in the resuscitation outcomes consortium population of 21 million. $J$ Am Coll Cardiol 55:1713-1720

17. Berdowski J, Blom MT, Bardai A, Tan HL, Tijssen JG, Koster RW (2011) Impact of onsite or dispatched automated external defibrillator use on survival after out-of-hospital cardiac arrest. Circulation 124:2225-2232

18. Nolan J, Soar J, Eikeland H (2006) The chain of survival. Resuscitation 71:270-271

19. Muller D, Agrawal R, Arntz HR (2006) How sudden is sudden cardiac death? Circulation 114:1146-1150

20. Waalewijn RA, Tijssen JG, Koster RW (2001) Bystander initiated actions in out-of-hospital cardiopulmonary resuscitation: results from the Amsterdam Resuscitation Study (ARRESUST). Resuscitation 50:273-279

21. Sasson C, Rogers MA, Dahl J, Kellermann AL (2010) Predictors of survival from out-of-hospital cardiac arrest: a systematic review and meta-analysis. Circ Cardiovasc Qual Outcomes 3:63-81

22. Nehme Z, Andrew E, Bernard S, Smith K (2015) Comparison of out-of-hospital cardiac arrest occurring before and after paramedic arrival epidemiology, survival to hospital discharge and 12-month functional recovery. Resuscitation 89:50-57

23. Takei Y, Nishi T, Kamikura T et al (2015) Do early emergency calls before patient collapse improve survival after out-of-hospital cardiac arrests? Resuscitation 88:20-27

24. Valenzuela TD, Roe DJ, Cretin S, Spaite DW, Larsen MP (1997) Estimating effectiveness of cardiac arrest interventions: a logistic regression survival model. Circulation 96:3308-3313

25. Holmberg M, Holmberg S, Herlitz J, Gardelov B (1998) Survival after cardiac arrest outside hospital in Sweden. Swedish Cardiac Arrest Registry. Resuscitation 36:29-36

26. Holmberg M, Holmberg S, Herlitz J (2001) Factors modifying the effect of bystander cardiopulmonary resuscitation on survival in out-of-hospital cardiac arrest patients in Sweden. Eur Heart J 22:511-519

27. Wissenberg M, Lippert FK, Folke F et al (2013) Association of national initiatives to improve cardiac arrest management with rates of bystander intervention and patient survival after out-ofhospital cardiac arrest. JAMA 310:1377-1384

28. Hasselqvist-Ax I, Riva G, Herlitz J et al (2015) Early cardiopulmonary resuscitation in out-of-hospital cardiac arrest. N Engl J Med 372:2307-2315

29. Rea TD, Fahrenbruch C, Culley L et al (2010) CPR with chest compresssions alone or with rescue breathing. NEngl J Med 363:423-433

30. Svensson L, Bohm K, Castren M et al (2010) Compression-only CPR or standard CPR in out-ofhospital cardiac arrest. NEngl J Med 363:434-442

31. Hupfl M, Selig HF, Nagele P (2010) Chestcompression-only versus standard cardiopulmonary resuscitation: a meta-analysis. Lancet 376:1552-1557

32. Valenzuela TD, Roe DJ, Nichol G, Clark LL, Spaite DW, Hardman RG (2000) Outcomes of rapid defibrillation by security officers after cardiac arrest in casinos. NEngl J Med 343:1206-1209

33. Ringh M, Rosenqvist M, Hollenberg J et al (2015) Mobile-phone dispatch of laypersons for CPR in out-of-hospital cardiac arrest. N Engl J Med 372:2316-2325

34. Larsen MP, Eisenberg MS, Cummins RO, Hallstrom AP (1993) Predicting survival from out-of-hospital cardiac arrest: a graphic model. Ann Emerg Med 22:1652-1658

35. Nolan JP, Soar J, Cariou A et al (2015) European Resuscitation Council Guidelines for Resuscitation 2015 Section 5 Post Resuscitation Care. Resuscitation 95:201-221

36. van Alem AP, Vrenken RH, de Vos R, Tijssen JG, Koster RW (2003) Use of automated external defibrillator by first responders in out of hospital cardiac arrest: prospective controlled trial. BMJ 327:1312

37. Fothergill RT, Watson LR, Chamberlain D, Virdi GK, Moore FP, Whitbread M (2013) Increases in survival from out-of-hospital cardiac arrest: a five year study. Resuscitation 84:1089-1092

38. Perkins GD, Lall R, Quinn T et al (2015) Mechanical versus manual chest compression for out-ofhospital cardiac arrest (PARAMEDIC): a pragmatic, cluster randomised controlled trial. Lancet 385:947-955

39. Weisfeldt ML, Becker LB (2002) Resuscitation after cardiac arrest: a 3-phase time-sensitive model. JAMA 288:3035-3038

40. Zijlstra JA, Stieglis R, Riedijk F, Smeekes M, van der Worp WE, Koster RW (2014) Local lay rescuers with AEDs, alerted by text messages, contribute to early defibrillation in a Dutch out-of-hospital cardiac arrest dispatch system. Resuscitation 85:1444-1449

41. Kerber RE, Becker LB, Bourland JD et al (1997) Automatic external defibrillators for public access defibrillation: recommendations for specifying and reporting arrhythmia analysis algorithm performance, incorporating new waveforms, and enhancing safety. A statement for health 
professionals from the American Heart Association Task Force on Automatic External Defibrillation, Subcommittee on AED Safety and Efficacy. Circulation 95:1677-1682

42. Calle PA, Mpotos N, Calle SP, Monsieurs KG (2015) Inaccurate treatment decisions of automated external defibrillators used by emergency medical services personnel: incidence, cause and impact on outcome. Resuscitation 88:68-74

43. Bahr J, Klingler H, PanzerW, Rode H, Kettler D (1997) Skills of lay people in checking the carotid pulse. Resuscitation 35:23-26

44. Nyman J, Sihvonen M (2000) Cardiopulmonary resuscitation skills in nurses and nursing students. Resuscitation 47:179-184

45. Tibballs J, Russell P (2009) Reliability of pulse palpation by healthcare personnel to diagnose paediatric cardiac arrest. Resuscitation 80:61-64

46. Tibballs J, Weeranatna C (2010) The influence of time on the accuracy of healthcare personnel to diagnose paediatric cardiac arrest by pulse palpation. Resuscitation 81:671-675

47. Moule P (2000) Checking the carotid pulse: diagnostic accuracy in students of the healthcare professions. Resuscitation 44:195-201

48. BobrowBJ,ZuercherM, Ewy GA etal (2008) Gasping during cardiac arrest in humans is frequent and associated with improved survival. Circulation 118:2550-2554

49. Perkins GD, Stephenson B, Hulme J, Monsieurs KG (2005) Birmingham assessment of breathing study (BABS). Resuscitation 64:109-113

50. Perkins GD, Walker G, Christensen K, Hulme J, Monsieurs KG (2006) Teaching recognition of agonal breathing improves accuracy of diagnosing cardiac arrest. Resuscitation 70:432-437

51. Breckwoldt J, Schloesser S, Arntz HR (2009) Perceptions of collapse and assessment of cardiac arrest by bystanders of out-of-hospital cardiac arrest (OOHCA). Resuscitation 80:1108-1113

52. Stecker EC, Reinier K, Uy-Evanado A et al (2013) Relationship between seizure episode and sudden cardiac arrest in patients with epilepsy: a community-based study. Circ Arrhythm Electrophysiol 6:912-916

53. Kuisma M, Boyd J, Vayrynen T, Repo J, Nousila-Wiik $M$, Holmstrom P (2005) Emergency call processing and survival from out-of-hospital ventricular fibrillation. Resuscitation 67:89-93

54. Berdowski J, Beekhuis F, Zwinderman AH, Tijssen JG, Koster RW (2009) Importance of the first link: description and recognition of an out-of-hospital cardiac arrest in an emergency call. Circulation 119:2096-2102

55. Heward A, Damiani M, Hartley-Sharpe C (2004) Does the use of the Advanced Medical Priority Dispatch System affect cardiac arrest detection? Emerg Med J 21:115-118

56. Eisenberg MS, Hallstrom AP, Carter WB, Cummins RO, Bergner L, Pierce J (1985) Emergency CPR instruction via telephone. Am J Public Health 75:47-50

57. Stipulante S, Tubes R, El Fassi M et al (2014) Implementation of the ALERT algorithm, a new dispatcher-assisted telephone cardiopulmonary resuscitation protocol, in non-Advanced Medical Priority Dispatch System (AMPDS) Emergency Medical Services centres. Resuscitation 85:177-181

58. Castren M, Kuisma M, Serlachius J, Skrifvars M (2001) Do health care professionals report sudden cardiac arrest better than laymen? Resuscitation 51:265-268
59. Hallstrom AP, Cobb LA, Johnson E, Copass MK (2003) Dispatcher assisted CPR: implementation and potential benefit. A 12-year study. Resuscitation 57:123-129

60. Dami F, Fuchs V, Praz L, Vader JP (2010) Introducing systematic dispatcher-assisted cardiopulmonary resuscitation (telephone-CPR) in a non-Advanced Medical Priority Dispatch System (AMPDS): implementation process and costs. Resuscitation 81:848-852

61. Nurmi J, Pettila V, Biber B, Kuisma M, Komulainen R, Castren M (2006) Effect of protocol compliance to cardiac arrest identification by emergency medical dispatchers. Resuscitation 70:463-469

62. Lewis M, Stubbs BA, Eisenberg MS (2013) Dispatcher-assisted cardiopulmonary resuscitation: time to identify cardiac arrest and deliver chest compression instructions. Circulation 128:1522-1530

63. Hauff SR, Rea TD, Culley LL, Kerry F, Becker L, Eisenberg MS (2003) Factors impeding dispatcherassisted telephone cardiopulmonary resuscitation. Ann Emerg Med 42:731-737

64. Bohm K, Stalhandske B, Rosenqvist M, Ulfvarson J, Hollenberg J, Svensson L (2009) Tuition of emergency medical dispatchers in the recognition ofagonal respiration increases the use oftelephone assisted CPR. Resuscitation 80:1025-1028

65. Bohm K, Rosenqvist M, Hollenberg J, Biber B, Engerstrom L, Svensson L (2007) Dispatcherassisted telephone-guided cardiopulmonary resuscitation: an underused lifesaving system. Eur JEmerg Med 14:256-259

66. Bång A, Herlitz J, Martinell S (2003) Interaction between emergency medical dispatcher and caller in suspected out-of-hospital cardiac arrest calls with focus on agonal breathing. A review of 100 tape recordings of true cardiac arrest cases. Resuscitation 56:25-34

67. Roppolo LP, Westfall A, Pepe PE et al (2009) Dispatcher assessments for agonal breathing improve detection of cardiac arrest. Resuscitation 80:769-772

68. Tanaka $Y$, Taniguchi J, Wato $Y$, Yoshida $Y$, Inaba $H$ (2012) The continuous quality improvement project for telephone-assisted instruction of cardiopulmonary resuscitation increased the incidence of bystander CPR and improved the outcomes of out-of-hospital cardiac arrests. Resuscitation 83:1235-1241

69. Clawson J, Olola C, Heward A, Patterson B (2007) Cardiac arrest predictability in seizure patients based on emergency medical dispatcher identification of previous seizure or epilepsy history. Resuscitation 75:298-304

70. Akahane M, Ogawa T, Tanabe S et al (2012) Impact of telephone dispatcher assistance on the outcomes of pediatric out-of-hospital cardiac arrest. Crit Care Med 40:1410-1416

71. Bray JE, Deasy C, Walsh J, Bacon A, Currell A, Smith K (2011) Changing EMS dispatcher CPR instructions to 400 compressions before mouth-to-mouth improved bystander CPR rates. Resuscitation 82:1393-1398

72. Culley LL, Clark JJ, Eisenberg MS, Larsen MP (1991) Dispatcher-assisted telephone CPR: common delays and time standards for delivery. Ann Emerg Med 20:362-366

73. Rea TD, Eisenberg MS, Culley LL, Becker L (2001) Dispatcher-assisted cardiopulmonary resuscitation and survival in cardiac arrest. Circulation 104:2513-2516

74. Hallstrom AP (2000) Dispatcher-assisted "phone“ cardiopulmonary resuscitation by chest compres- sion alone or with mouth-to-mouth ventilation. Crit Care Med 28:N190-N192

75. Stromsoe A, Svensson $L$, Axelsson $A B$ et al (2015) Improved outcome in Sweden after outof-hospital cardiac arrest and possible association with improvements in every link in the chain of survival. Eur Heart J 36:863-871

76. Takei $\mathrm{Y}$, Inaba H, Yachida T, Enami M, Goto Y, Ohta K (2010) Analysis of reasons for emergency call delays in Japan in relation to location: high incidence of correctable causes and the impact of delays on patient outcomes. Resuscitation 81:1492-1498

77. Herlitz J, Engdahl J, Svensson L, Young M, Angquist KA, Holmberg S (2003) A short delay from out of hospital cardiac arrest to call for ambulance increases survival. Eur Heart J 24:1750-1755

78. Nehme Z, Andrew E, Cameron P et al (2014) Direction of first bystander call for help is associated with outcome from out-of-hospital cardiac arrest. Resuscitation 85:42-48

79. Birkenes TS, Myklebust $\mathrm{H}$, Neset A, Olasveengen TM, Kramer-Johansen J (2012) Video analysis of dispatcher-rescuer teamwork-Effects on CPR technique and performance. Resuscitation 83:494-499

80. Birkenes TS, Myklebust H, Kramer-Johansen J (2013) Time delays and capability of elderly to activate speaker function for continuous telephone CPR. Scand J Trauma Resusc Emerg Med 21:40

81. Marsch S, Tschan F, Semmer NK, Zobrist R, Hunziker PR, Hunziker S (2013) ABC versus CAB for cardiopulmonary resuscitation: a prospective, randomized simulator-based trial. Swiss Med Wkly 143:w13856

82. Lubrano R, Cecchetti C, Bellelli E et al (2012) Comparison of times of intervention during pediatric $C P R$ maneuvers using $A B C$ and $C A B$ sequences: a randomized trial. Resuscitation 83:1473-1477

83. Sekiguchi H, Kondo Y, Kukita I (2013) Verification of changes in the time taken to initiate chest compressions according to modified basic life support guidelines. Am JEmerg Med 31:1248-1250

84. Kobayashi M, Fujiwara A, Morita H et al (2008) A manikin-based observational study on cardiopulmonary resuscitation skills at the Osaka Senri medical rally. Resuscitation 78:333-339

85. Cha KC, Kim HJ, Shin HJ, Kim H, Lee KH, Hwang SO (2013) Hemodynamic effect of external chest compressions at the lower end of the sternum in cardiac arrest patients. J Emerg Med 44:691-697

86. Qvigstad E, Kramer-Johansen J, Tomte 0 et al (2013) Clinical pilot study of different hand positions during manual chest compressions monitored with capnography. Resuscitation 84:1203-1207

87. Orlowski JP (1986) Optimum position for external cardiac compression in infants and young children. Ann Emerg Med 15:667-673

88. Chamberlain D, Smith A, Colquhoun M, Handley AJ, Kern KB, Woollard M (2001) Randomised controlled trials of staged teaching for basic life support: 2. Comparison of CPR performance and skill retention using either staged instruction or conventional training. Resuscitation 50:27-37

89. Handley AJ (2002) Teaching hand placement for chest compression-a simpler technique. Resuscitation 53:29-36

90. Handley AJ, Handley JA (2004) Performing chest compressions in a confined space. Resuscitation 61:55-61

91. Perkins GD, Stephenson BT, Smith CM, Gao F (2004) A comparison between over-the-head 
and standard cardiopulmonary resuscitation. Resuscitation 61:155-161

92. Hostler D, Everson-Stewart S, Rea TD et al (2011) Effect of real-time feedback during cardiopulmonary resuscitation outside hospital: prospective, cluster-randomised trial. BMJ342:d512

93. Stiell IG, Brown SP, Christenson J et al (2012) What is the role of chest compression depth during out-ofhospital cardiac arrest resuscitation? Crit Care Med 40:1192-1198

94. Stiell IG, Brown SP, Nichol G et al (2014) What is the optimal chest compression depth during outof-hospital cardiac arrest resuscitation of adult patients? Circulation 130:1962-1970

95. Vadeboncoeur T, Stolz U, Panchal A et al (2014) Chest compression depth and survival in out-ofhospital cardiac arrest. Resuscitation 85:182-188

96. Hellevuo H, Sainio M, Nevalainen Ret al (2013) Deeper chest compression - more complications for cardiac arrest patients? Resuscitation 84:760-765

97. Idris AH, Guffey D, Pepe PE et al (2015) Chest compression rates and survival following out-ofhospital cardiac arrest. Crit Care Med 43:840-848

98. Idris AH, Guffey D, Aufderheide TP et al (2012) Relationship between chest compression rates and outcomes from cardiac arrest. Circulation 125:3004-3012

99. Cheskes S, Schmicker RH, Verbeek PR et al (2014) The impact of peri-shock pause on survival from out-of-hospital shockable cardiac arrest during the Resuscitation Outcomes Consortium PRIMED trial. Resuscitation 85:336-342

100. Cheskes S, Schmicker RH, Christenson J et al (2011) Perishock pause: an independent predictor of survival from out-of-hospital shockable cardiac arrest. Circulation 124:58-66

101. Vaillancourt C, Everson-Stewart S, Christenson $J$ et al (2011) The impact of increased chest compression fraction on return of spontaneous circulation for out-of-hospital cardiac arrest patients not in ventricular fibrillation. Resuscitation 82:1501-1507

102. Sell RE, Sarno R, Lawrence B et al (2010) Minimizing pre- and post-defibrillation pauses increases the likelihood of return of spontaneous circulation (ROSC). Resuscitation 81:822-825

103. Christenson J, Andrusiek D, Everson-Stewart S et al (2009) Chest compression fraction determines survival in patients with out-of-hospital ventricular fibrillation. Circulation 120:1241-1247

104. Delvaux AB, Trombley MT, Rivet CJ et al (2009) Design and development of a cardiopulmonary resuscitation mattress. J Intensive Care Med 24:195-199

105. Nishisaki A, Maltese MR, Niles DE et al (2012) Backboards are important when chest compressions are provided on a soft mattress. Resuscitation 83:1013-1020

106. Sato H, Komasawa N, Ueki R et al (2011) Backboard insertion in the operating table increases chest compression depth: a manikin study. J Anesth 25:770-772

107. Perkins GD, Smith CM, Augre C et al (2006) Effects of a backboard, bed height, and operator position on compression depth during simulated resuscitation. Intensive Care Med 32:1632-1635

108. Perkins GD, Kocierz L, Smith SC, McCulloch RA, Davies RP (2009) Compression feedback devices over estimate chest compression depth when performed on a bed. Resuscitation 80:79-82

109. Cloete G, Dellimore KH, Scheffer C, Smuts MS, Wallis LA (2011) The impact of backboard size and orientation on sternum-to-spine compression depth and compression stiffness in a manikin study of CPR using two mattress types. Resuscitation 82:1064-1070

110. Niles DE, Sutton RM, Nadkarni VM et al (2011) Prevalence and hemodynamic effects of leaning during CPR. Resuscitation 82(Suppl 2):23-26

111. Fried DA, Leary $M$, Smith DA et al (2011) The prevalence of chest compression leaning during in-hospital cardiopulmonary resuscitation. Resuscitation 82:1019-1024

112. Zuercher M, Hilwig RW, Ranger-Moore J et al (2010) Leaning during chest compressions impairs cardiac output and left ventricular myocardial blood flow in piglet cardiac arrest. Crit Care Med 38:1141-1146

113. Aufderheide TP, Pirrallo RG, Yannopoulos D et al (2005) Incomplete chest wall decompression: a clinical evaluation of CPR performance by EMS personnel and assessment of alternative manual chest compression-decompression techniques. Resuscitation 64:353-362

114. Yannopoulos D, McKnite S, Aufderheide TP et al (2005) Effects of incomplete chest wall decompression during cardiopulmonary resuscitation on coronary and cerebral perfusion pressures in a porcine model of cardiac arrest. Resuscitation 64:363-372

115. Jung $E$, Babbs CF, Lenhart S, Protopopescu VA (2006) Optimal strategy for cardiopulmonary resuscitation with continuous chest compression. Acad Emerg Med 13:715-721

116. Betz AE, Menegazzi JJ, Logue ES, Callaway CW, Wang HE (2006) A randomized comparison of manual, mechanical and high-impulse chest compression in a porcine model of prolonged ventricular fibrillation. Resuscitation 69:495-501

117. Koeken $Y$, Aelen P, Noordergraaf GJ, Paulussen I, Woerlee P, Noordergraaf A (2011) The influence of nonlinear intra-thoracic vascular behaviour and compression characteristics on cardiac output during CPR. Resuscitation 82:538-544

118. Sunde K, Wik L, Naess PA, llebekk A, Nicolaysen $G$, Steen PA (1998) Effect of different compression-decompression cycles on haemodynamics during ACD-CPR in pigs. Resuscitation 36:123-13

119. Handley AJ, Handley JA (1995) The relationship between rate of chest compression and compres sion:relaxation ratio. Resuscitation 30:237-241

120. Swart GL, Mateer JR, DeBehnke DJ, Jameson SJ, Osborn JL (1994) The effect of compression duration on hemodynamics during mechanical high-impulse CPR. Acad Emerg Med 1:430-437

121. Dean JM, Koehler RC, Schleien CL et al (1991) Improved blood flow during prolonged cardiopulmonary resuscitation with $30 \%$ duty cycle in infant pigs. Circulation 84:896-904

122. Halperin HR, Tsitlik JE, Guerci AD et al (1986) Determinants of blood flow to vital organs during cardiopulmonary resuscitation in dogs. Circulation 73:539-50

123. Fitzgerald KR, Babbs CF, Frissora HA, Davis RW, Silver DI (1981) Cardiac output during cardiopulmonary resuscitation at various compression rates and durations. Am JPhysiol 241:H442-H448

124. Johnson B, Coult J, Fahrenbruch C et al (2015) Cardiopulmonary resuscitation duty cycle in outof-hospital cardiac arrest. Resuscitation 87:86-90

125. Yeung J, Meeks R, Edelson D, Gao F, Soar J, Perkins GD (2009) The use of CPRfeedback/prompt devices during training and CPR performance: a systematic review. Resuscitation 80:743-751

126. Kirkbright S, Finn J, Tohira H, Bremner A, Jacobs I, Celenza A (2014) Audiovisual feedback device use by health care professionals during CPR: a systema- tic review and meta-analysis of randomised and non-randomised trials. Resuscitation 85:460-471

127. Bohn A, Weber TP, Wecker S et al (2011) The addition of voice prompts to audiovisual feedback and debriefing does not modify CPR quality or outcomes in out of hospital cardiac arrest-a prospective, randomized trial. Resuscitation 82:257-262

128. Abella BS, Edelson DP, Kim S et al (2007) CPR quality improvement during in-hospital cardiac arrest using a real-time audiovisual feedback system. Resuscitation 73:54-61

129. Berg RA, Sanders AB, Milander M, Tellez D, Liu P, Beyda D (1994) Efficacy of audio-prompted rate guidance in improving resuscitator performance of cardiopulmonary resuscitation on children. Acad Emerg Med 1:35-40

130. Bobrow BJ, Vadeboncoeur TF, Stolz $U$ et al (2013) The influence of scenario-based training and real-time audiovisual feedback on out-ofhospital cardiopulmonary resuscitation quality and survival from out-of-hospital cardiac arrest Ann Emerg Med 62:47-56.e1

131. Chiang WC, Chen WJ, Chen SY et al (2005) Better adherence to the guidelines during cardiopulmonary resuscitation through the provision of audioprompts. Resuscitation 64:297-301

132. Kern KB, Sanders AB, Raife J, Milander MM, Otto CW, Ewy GA (1992) A study of chest compression rates during cardiopulmonary resuscitation in humans: the importance of rate-directed chest compressions. Arch Intern Med 152:145-149

133. Kramer-Johansen J, Myklebust $H$, Wik L et al (2006) Quality of out-of-hospital cardiopulmonary resuscitation with real time automated feedback: a prospective interventional study. Resuscitation 71:283-292

134. Lukas RP, Grasner JT, Seewald S et al (2012) Chest compression quality management and return of spontaneous circulation: a matched-pair registry study. Resuscitation 83:1212-1218

135. Niles D, Nysaether J, Sutton R et al (2009) Leaning is common during in-hospital pediatric CPR, and decreased with automated corrective feedback. Resuscitation 80:553-557

136. Sainio M, Kamarainen A, Huhtala Hetal (2013) Realtime audiovisual feedback system in a physicianstaffed helicopter emergency medical service in Finland: the quality results and barriers to implementation. Scand J Trauma Resusc Emerg Med 21:50

137. Sutton RM, Niles D, French B et al (2014) First quantitative analysis of cardiopulmonary resuscitation quality during in-hospital cardiac arrests of young children. Resuscitation 85:70-74

138. Couper K, Kimani P, Abella BS, Chilwan M, Cooke MW, Davies RP (2015) The System-Wide Effect of Real-Time Audiovisual Feedback and Postevent Debriefing for In-Hospital Cardiac Arrest: the Cardiopulmonary Resuscitation Quality Improvement Initiative. Crit Care Med. http:// dx.doi.org/10.1097/CCM.0000000000001202 (im Druck)

139. Couper K, Salman B, Soar J, Finn J, Perkins GD (2013) Debriefing to improve outcomes from critical illness: a systematic review and meta-analysis. Intensive Care Med 39:1513-1523

140. Kern KB, Hilwig RW, Berg RA, Sanders AB, Ewy GA (2002) Importance of continuous chest compressions during cardiopulmonary resuscitation: improved outcome during a simulated single layrescuer scenario. Circulation 105:645-649

141. Deakin CD, O'Neill JF, Tabor T (2007) Does compression-only cardiopulmonary resuscitation 


\section{ERC Leitlinien}

generate adequate passive ventilation during cardiac arrest? Resuscitation 75:53-59

142. Bobrow BJ, Clark LL, Ewy GA et al (2008) Minimally interrupted cardiac resuscitation by emergency medical services for out-of-hospital cardiac arrest. JAMA 299:1158-1165

143. Idris A, Wenzel V, Banner MJ, Melker RJ (1995) Smaller tidal volumes minimize gastric inflation during CPR with an unprotected airway. Circulation 92(suppl):I-759

144. Winkler M, Mauritz W, Hackl W et al (1998) Effects of half the tidal volume during cardiopulmonary resuscitation on acid-base balance and haemodynamics in pigs. Eur J Emerg Med 5:201-206

145. Idris A, Gabrielli A, Caruso L (1999) Smaller tidal volume is safe and effective for bagvalve-ventilation, but not for mouth-to-mouth ventilation: an animal model for basic life support. Circulation 100:I-644

146. DorphE, WikL, Steen PA (2004) Arterial blood gases with $700 \mathrm{ml}$ tidal volumes during out-of-hospital CPR. Resuscitation 61:23-27

147. Wenzel V, Idris AH, Banner MJ, Kubilis PS, Williams JLJ (1998) Influence of tidal volume on the distribution of gas between the lungs and stomach in the nonintubated patient receiving positivepressure ventilation. Crit Care Med 26:364-368

148. von Goedecke A, Wagner-Berger HG, Stadlbauer $\mathrm{KH}$ et al (2004) Effects of decreasing peak flow rate on stomach inflation during bag-valve-mask ventilation. Resuscitation 63:131-136

149. Aufderheide TP, Sigurdsson G, Pirrallo RG et al (2004) Hyperventilation-induced hypotension during cardiopulmonary resuscitation. Circulation 109:1960-1965

150. O'Neill JF, Deakin CD (2007) Do we hyperventilate cardiacarrest patients? Resuscitation 73:82-85

151. Gazmuri RJ, Ayoub IM, Radhakrishnan J, Motl J, Upadhyaya MP (2012) Clinically plausible hyperventilation does not exert adverse hemodynamic effects during CPR but markedly reduces end-tidal PCO(2). Resuscitation 83:259-264

152. Baskett P, Nolan J, Parr M (1996) Tidal volumes which are perceived to be adequate for resuscitation. Resuscitation 31:231-234

153. Beesems SG, Wijmans L, Tijssen JG, Koster RW (2013) Duration of ventilations during cardiopulmonary resuscitation by lay rescuers and first responders: relationship between delivering chest compressions and outcomes. Circulation 127:1585-1590

154. Ruben $H$ (1964) The immediate treatment of respiratory failure. Br J Anaesth 36:542-549

155. Kowalik MM (2007) Mouth-to-tracheostomy tube ventilation in an emergency situation. Resuscitation 73:322-323

156. Sanders AB, Kern KB, Berg RA, Hilwig RW, Heidenrich J, Ewy GA (2002) Survival and neurologic outcome after cardiopulmonary resuscitation with four different chest compression-ventilation ratios. Ann Emerg Med 40:553-562

157. Dorph E, Wik L, Stromme TA, Eriksen M, Steen PA (2003) Quality of CPR with three different ventilation:compression ratios. Resuscitation 58:193-201

158. Dorph E, Wik L, Stromme TA, Eriksen M, Steen PA (2004) Oxygen delivery and return of spontaneous circulation with ventilation:compression ratio 2:30 versus chest compressions only CPR in pigs. Resuscitation 60:309-318

159. Babbs CF, Kern KB (2002) Optimum compression to ventilation ratios in CPR under realistic, practical conditions: a physiological and mathematical analysis. Resuscitation 54:147-157

160. Fenici $P$, Idris $A H$, Lurie $K G$, Ursella $S$, Gabrielli $A$ (2005) What is the optimal chest compressionventilation ratio? Curr Opin Crit Care 11:204-211

161. Sayre MR, Cantrell SA, White LJ, Hiestand BC, Keseg DP, Koser S (2009) Impact of the 2005 American Heart Association cardiopulmonary resuscitation and emergency cardiovascular care guidelines on out-of-hospital cardiac arrest survival. Prehos Emerg Care 13:469-477

162. Olasveengen TM, VikE, Kuzovlev A, Sunde K (2009) Effect of implementation of new resuscitation guidelines on quality of cardiopulmonary resuscitation and survival. Resuscitation 80:407-411

163. Aufderheide TP, Lurie KG (2004) Death by hyperventilation: a common and life-threatening problem during cardiopulmonary resuscitation. Crit Care Med 32:345-351

164. Steinmetz J, Barnung S, Nielsen SL, Risom M, Rasmussen LS (2008) Improved survival after an out-of-hospital cardiac arrest using new guidelines. Acta Anaesthesiol Scand 52:908-913

165. Hinchey PR, Myers JB, Lewis R et al (2010) Improved out-of-hospital cardiac arrest survival after the sequential implementation of 2005 AHA guidelines for compressions, ventilations, and induced hypothermia: the Wake County experience. Ann Emerg Med 56:348-357

166. Chandra NC, Gruben KG, Tsitlik JE et al (1994) Observations of ventilation during resuscitation in a canine model. Circulation 90:3070-3075

167. Turner I, Turner S, Armstrong V (2002) Does the compression to ventilation ratio affect the quality of CPR: a simulation study. Resuscitation 52:55-62

168. Geddes LA, Rundell A, Otlewski M, Pargett M (2008) How much lung ventilation is obtained with only chest-compression CPR? Cardiovasc Eng 8:145-148

169. Berg RA, Kern KB, Hilwig RW et al (1997) Assisted ventilation does not improve outcome in a porcine model of single-rescuer bystander cardiopulmonary resuscitation. Circulation 95:1635-1641

170. Berg RA, Kern KB, Hilwig RW, Ewy GA (1997) Assisted ventilation during ,bystander' $\mathrm{CPR}$ in a swine acute myocardial infarction model does not improve outcome. Circulation 96:4364-4371

171. Panchal AR, BobrowBJ, Spaite DWetal (2013)Chest compression-only cardiopulmonary resuscitation performed by lay rescuers for adult out-of-hospital cardiac arrest due to non-cardiac aetiologies. Resuscitation 84:435-439

172. Kitamura T, Iwami T, Kawamura T et al (2011) Timedependent effectiveness of chest compressiononly and conventional cardiopulmonary resuscitation for out-of-hospital cardiac arrest of cardiac origin. Resuscitation 82:3-9

173. Mohler MJ, Wendel CS, Mosier J et al (2011) Cardiocerebral resuscitation improves out-ofhospital survival in older adults. J Am Geriatr Soc 59:822-826

174. Bobrow BJ, Spaite DW, Berg RA et al (2010) Chest compression-only CPR by lay rescuers and survival from out-of-hospital cardiac arrest. JAMA 304:1447-1454

175. Kitamura T, Iwami T, Kawamura T, Nagao K, Tanaka $\mathrm{H}$, Hiraide A (2010) Bystander-initiated rescue breathing for out-of-hospital cardiac arrests of noncardiac origin. Circulation 122:293-299

176. Ong ME, Ng FS, Anushia P et al (2008) Comparison of chest compression only and standard cardiopulmonary resuscitation for out-of-hospital cardiac arrest in Singapore. Resuscitation 78:119-126
177. Bohm K, Rosenqvist M, Herlitz J, Hollenberg J, Svensson L (2007) Survival is similar after standard treatment and chest compression only in out-ofhospital bystander cardiopulmonary resuscitation. Circulation 116:2908-2912

178. SOS-KANTO Study Group (2007) Cardiopulmonary resuscitation by bystanders with chest compression only (SOS-KANTO): an observational study. Lancet 369:920-926

179. Iwami T, Kawamura T, Hiraide A et al (2007) Effectiveness of bystander-initiated cardiac-only resuscitation for patients with out-of-hospital cardiac arrest. Circulation 116:2900-2907

180. Bossaert L, Van Hoeyweghen R(1989) Evaluation of cardiopulmonary resuscitation (CPR) techniques. The Cerebral Resuscitation Study Group. Resuscitation 17(Suppl):99-109. (discussion S99-206)

181. Gallagher EJ, Lombardi G, Gennis P (1995) Effectiveness of bystander cardiopulmonary resuscitation and survival following out-ofhospital cardiac arrest. JAMA 274:1922-1925

182. Olasveengen TM, Wik L, Steen PA (2008) Standard basic life support vs. continuous chest compressions only in out-of-hospital cardiac arrest. Acta Anaesthesiol Scand 52:914-919

183. Kitamura T, Iwami T, Kawamura T et al (2010) Conventional and chest-compression-only cardiopulmonary resuscitation by bystanders for children who have out-of-hospital cardiac arrests: a prospective, nationwide, population-based cohort study. Lancet 375:1347-1354

184. Goto Y, Maeda T, Goto Y (2014) Impact of dispatcher-assisted bystander cardiopulmonary resuscitation on neurological outcomes in children with out-of-hospital cardiac arrests: a prospective, nationwide, population-based cohort study. J Am Heart Assoc 3:e000499

185. Yeung J, Okamoto D, Soar J, Perkins GD (2011) AED training and its impact on skill acquisition, retention and performance-a systematic review of alternative training methods. Resuscitation 82:657-664

186. Edelson DP, Abella BS, Kramer-Johansen J et al (2006) Effects of compression depth and pre-shock pauses predict defibrillation failure during cardiac arrest. Resuscitation 71:137-145

187. Mitani Y, Ohta K, Yodoya N et al (2013) Public access defibrillation improved the outcome after outof-hospital cardiac arrest in school-age children: a nationwide, population-based, Utstein registry study in Japan. Europace 15:1259-1266

188. Johnson MA, Grahan BJ, Haukoos JS et al (2014) Demographics, bystander CPR, and AED use in out-of-hospital pediatric arrests. Resuscitation 85:920-926

189. Akahane M, Tanabe S, Ogawa T et al (2013) Characteristics and outcomes of pediatric out-ofhospital cardiac arrest by scholastic age category. Pediatr Crit Care Med 14:130-136

190. Bar-Cohen Y, Walsh EP, Love BA, Cecchin F (2005) First appropriate use of automated external defibrillator in an infant. Resuscitation 67:135-137

191. Divekar A, Soni R (2006) Successful parental use of an automated external defibrillator for an infant with long-QT syndrome. Pediatrics 118:e526-e529

192. Rodriguez-Nunez A, Lopez-Herce J, Garcia C, Dominguez P, Carrillo A, Bellon JM (2006) Pediatric defibrillation after cardiac arrest: initial response and outcome. Crit Care 10:R113

193. Samson RA, Nadkarni VM, Meaney PA, Carey SM, Berg MD, Berg RA (2006) Outcomes of in-hospital ventricular fibrillation in children. $N$ Engl J Med 354:2328-2339 
194. Atkins DL, Everson-Stewart S, Sears GK et al (2009) Epidemiology and outcomes from out-of-hospital cardiac arrest in children: the Resuscitation Outcomes Consortium Epistry-Cardiac Arrest. Circulation 119:1484-1491

195. Bardai A, Berdowski J, van der Werf $C$ et al (2011) Incidence, causes, and outcomes of out-of-hospital cardiac arrest in children. A comprehensive, prospective, population-based study in the Netherlands. J Am Coll Cardiol 57:1822-1828

196. Cobb LA, Fahrenbruch CE, Walsh TR et al (1999) Influence of cardiopulmonary resuscitation prior to defibrillation in patients with out-of-hospital ventricular fibrillation. JAMA 281:1182-1188

197. Wik L, Hansen TB, Fylling F et al (2003) Delaying defibrillation to give basic cardiopulmonary resuscitation to patients with out-of-hospital ventricular fibrillation: a randomized trial. JAMA 289:1389-1395

198. Jacobs IG, Finn JC, Oxer HF, Jelinek GA (2005) CPR before defibrillation in out-of-hospital cardiac arrest: a randomized trial. Emerg Med Australas 17:39-45

199. Baker PW, Conway J, Cotton C et al (2008) Defibrillation or cardiopulmonary resuscitation first for patients with out-of-hospital cardiac arrests found by paramedics to be in ventricular fibrillation? A randomised control trial. Resuscitation 79:424-431

200. Stiell IG, Nichol G, Leroux BG et al (2011) Early versus later rhythm analysis in patients with out-ofhospital cardiac arrest. NEngl J Med 365:787-797

201. Rea T, Prince D, Morrison L et al (2014) Association between survival and early versus later rhythm analysis in out-of-hospital cardiac arrest: do agency-level factors influence outcomes? Ann Emerg Med 64:1-8

202. Monsieurs KG, Vogels C, Bossaert LL, Meert P, Calle PA (2005) A study comparing the usability of fully automatic versus semi-automatic defibrillation by untrained nursing students. Resuscitation 64:41-47

203. Hosmans TP, Maquoi I, Vogels C et al (2008) Safety of fully automatic external defibrillation by untrained lay rescuers in the presence of a bystander. Resuscitation 77:216-219

204. Weisfeldt ML, Everson-Stewart S, Sitlani C et al (2011) Ventricular tachyarrhythmias after cardiac arrest in public versus at home. $\mathrm{N}$ Engl J Med 364:313-321

205. CaffreySL, Willoughby PJ,PepePE, BeckerLB (2002) Public use of automated external defibrillators. N Engl J Med 347:1242-1247

206. Page RL, Hamdan MH, McKenas DK (1998) Defibrillation aboard a commercial aircraft Circulation 97:1429-1430

207. O'Rourke MF, Donaldson E, Geddes JS (1997) An airline cardiac arrest program. Circulation 96:2849-2853

208. The Public Access Defibrillation Trial Investigators (2004) Public-access defibrillation and survival after out-of-hospital cardiac arrest. N Engl J Med 351:637-646

209. Kitamura T, Iwami T, Kawamura T, Nagao K, Tanaka $\mathrm{H}$, Hiraide A (2010) Nationwide public-access defibrillation in Japan. NEngl J Med 362:994-1004

210. NicholG, Valenzuela T, RoeD, ClarkL, HusztiE, Wells GA (2003) Cost effectiveness of defibrillation by targeted responders in public settings. Circulation 108:697-703

211. Nichol G, Huszti E, Birnbaum A et al (2009) Costeffectiveness of lay responder defibrillation for out-of-hospital cardiac arrest. Ann Emerg Med 54:226-35.e1-e2

212. Folke F, Lippert FK, Nielsen SL et al (2009) Location of cardiac arrest in a city center: strategic placement of automated external defibrillators in public locations. Circulation 120:510-517

213. Chan TC, Li H, Lebovic G et al (2013) Identifying locations for public access defibrillators using mathematical optimization. Circulation 127:1801-1809

214. Folke F, Gislason GH, Lippert FK et al (2010) Differences between out-of-hospital cardiac arrest in residential and public locations and implications for public-access defibrillation. Circulation 122:623-630

215. Hansen CM, Lippert FK, Wissenberg $M$ et al (2014) Temporal trends in coverage of historical cardiac arrests using a volunteer-based network of automated external defibrillators accessible to laypersons and emergency dispatch centers. Circulation 130:1859-1867

216. van Alem AP, Dijkgraaf MG, Tijssen JG, Koster RW (2004) Health system costs of out-of-hospita cardiac arrest in relation to time to shock. Circulation 110:1967-1973

217. Berdowski J, Kuiper MJ, Dijkgraaf MG, Tijssen JG, Koster RW (2010) Survival and health care costs until hospital discharge of patients treated with onsite, dispatched or without automated external defibrillator. Resuscitation 81:962-967

218. Waalewijn RA, de Vos R, Tijssen JG, Koster RW (2001) Survival models for out-of-hospital cardiopulmonary resuscitation from the perspectives of the bystander, the first responder, and the paramedic. Resuscitation 51:113-122

219. Priori SG, Bossaert LL, Chamberlain DA et al (2004) Policy statement: ESC-ERC recommendations for the use of automated external defibrillators (AEDs) in Europe. Resuscitation 60:245-252

220. Bardy GH, Lee KL, Mark DB et al (2008) Home use of automated external defibrillators for sudden cardiac arrest. N Engl J Med 358:1793-1804

221. Truhlar A, Deakin CD, Soar J et al (2015) European Resuscitation Council Guidelines for Resuscitation 2015 Section 4 Cardiac Arrest in Special Circumstances. Resuscitation 95:147-200

222. ILCOR presents a universal AED sign. European Resuscitation Council, 2008. https://www.erc.edu/ index.php/newsltem/en/nid=204/. Zugegriffen: 28. Juni 2015

223. Zafari AM, Zarter SK, Heggen V et al (2004) A program encouraging early defibrillation results in improved in-hospital resuscitation efficacy. J Am Coll Cardiol 44:846-852

224. Destro A, Marzaloni M, Sermasi S, Rossi F (1996) Automatic external defibrillators in the hospital as well? Resuscitation 31:39-43

225. Kloppe C, Jeromin A, Kloppe A, Ernst M, Mugge A, Hanefeld C (2013) First responder for inhospital resuscitation: 5-year experience with an automated external defibrillator-based program.J Emerg Med 44:1077-1082

226. Forcina MS, Farhat AY, O'Neil WW, Haines DE (2009) Cardiac arrest survival after implementation of automated external defibrillator technology in the in-hospital setting. Crit Care Med 37:1229-1236

227. Smith RJ, Hickey BB, Santamaria JD (2009) Automated external defibrillators and survival after in-hospital cardiac arrest: early experience at an Australian teaching hospital. Crit Care Resusc 11:261-265

228. Smith RJ, Hickey BB, Santamaria JD (2011) Automated external defibrillators and in-hospital cardiac arrest: patient survival and device performance at an Australian teaching hospital. Resuscitation 82:1537-1542

229. Chan PS, Krumholz HM, Spertus JA et al (2010) Automated external defibrillators and survival after in-hospital cardiac arrest. JAMA 304:2129-2136

230. Gibbison B, Soar J (2011) Automated external defibrillator use for in-hospital cardiac arrest is not associated with improved survival. Evid Based Med 16:95-96

231. Nolan JP, Soar J, Smith GB et al (2014) Incidence and outcome of in-hospital cardiac arrest in the United Kingdom National Cardiac Arrest Audit. Resuscitation 85:987-992

232. De Regge M, Monsieurs KG, Vandewoude K, Calle PA (2012) Should we use automated external defibrillators in hospital wards? Acta Clin Belg 67:241-245

233. Chan PS, Krumholz HM, Nichol G, Nallamothu BK (2008) Delayed time to defibrillation after inhospital cardiac arrest. N Engl J Med 358:9-17

234. Spearpoint KG, Gruber PC, Brett SJ (2009) Impact of the Immediate Life Support course on the incidence and outcome of in-hospital cardiac arrest calls: an observational study over 6 years. Resuscitation 80:638-643

235. White L, Rogers J, Bloomingdale Met al (2010) Dispatcher-assisted cardiopulmonary resuscitation: risks for patients not in cardiac arrest. Circulation 121:91-97

236. Haley KB, Lerner EB, Pirrallo RG, Croft H, Johnson A Uihlein M (2011) The frequency and consequences of cardiopulmonary resuscitation performed by bystanders on patients who are not in cardiac arrest. Prehos Emerg Care 15:282-287

237. Moriwaki Y, Sugiyama M, Tahara Y et al (2012) Complications of bystander cardiopulmonary resuscitation for unconscious patients without cardiopulmonary arrest. J Emerg Trauma Shock 5:3-6

238. Hoke RS, Chamberlain D (2004) Skeletal chest injuries secondary to cardiopulmonary resuscitation. Resuscitation 63:327-338

239. Miller AC, Rosati SF, Suffredini AF, Schrump DS (2014) A systematic review and pooled analysis of CPR-associated cardiovascular and thoracic injuries. Resuscitation 85:724-731

240. Sullivan F, Avstreih D (2000) Pneumothorax during CPR training: case report and review of the CPR literature. Prehosp Disaster Med 15:64-69

241. Cheung W, Gullick J, Thanakrishnan G et al (2009) Injuries occurring in hospital staff attending medical emergency team (MET) calls-a prospective, observational study. Resuscitation 80:1351-1356

242. Peberdy MA, Ottingham LV, Groh WJ et al (2006) Adverse events associated with lay emergency response programs: the publicaccess defibrillation trial experience. Resuscitation 70:59-65

243. McDonald $\mathrm{CH}$, Heggie J, Jones $\mathrm{CM}$, Thorne $\mathrm{CJ}$, Hulme J (2013) Rescuer fatigue under the 2010 ERC guidelines, and its effect on cardiopulmonary resuscitation (CPR) performance. Emerg Med J 30:623-627

244. Sugerman NT, Edelson DP, Leary M et al (2009) Rescuer fatigue during actual in-hospital cardiopulmonary resuscitation with audiovisual feedback: a prospective multicenter study. Resuscitation 80:981-984

245. Hoke RS, Heinroth K, Trappe HJ, Werdan K (2009) Is external defibrillation an electric threat for bystanders? Resuscitation 80:395-401

246. Sullivan JL, Chapman FW (2012) Will medical examination gloves protect rescuers from defibrillation voltages during hands-on defibrillation? Resuscitation 83:1467-1472 
247. Petley GW, Cotton AM, Deakin CD (2012) Handson defibrillation: theoretical and practical aspects of patient and rescuer safety. Resuscitation 83:551-556

248. Deakin CD, Lee-Shrewsbury V, Hogg K, Petley GW (2013) Do clinical examination gloves provide adequate electrical insulation for safe handson defibrillation? I: resistive properties of nitrile gloves. Resuscitation 84:895-899

249. Petley GW, Deakin CD (2013) Do clinical examination gloves provide adequate electrical insulation for safe hands-on defibrillation? II: material integrity following exposure to defibrillation waveforms. Resuscitation 84:900-903

250. Axelsson A, Herlitz J, Ekstrom L, Holmberg S (1996) Bystander-initiated cardiopulmonary resuscitation out-of-hospital. A first description of the bystanders and their experiences. Resuscitation 33:3-11

251. Axelsson A, Herlitz J, Karlsson T et al (1998) Factors surrounding cardiopulmonary resuscitation influencing bystanders' psychological reactions. Resuscitation 37:13-20

252. Jabre P, Belpomme V, Azoulay E et al (2013) Family presence during cardiopulmonary resuscitation. $\mathrm{N}$ Engl J Med 368:1008-1018

253. Jabre P, Tazarourte K, Azoulay E et al (2014) Offering the opportunity for family to be present during cardiopulmonary resuscitation: 1-year assessment. Intensive Care Med 40:981-987

254. Compton S, Fernandez R (2014) Presence during cardiopulmonary resuscitation is beneficial to family members in the out-of-hospital setting. Evid Based Med 19:13

255. Bierens JJ, Berden HJ (1996) Basic-CPR and AIDS: are volunteer life-savers prepared for a storm? Resuscitation 32:185-191

256. Mejicano GC, Maki DG (1998) Infections acquired during cardiopulmonary resuscitation: estimating the risk and defining strategies for prevention. Ann Intern Med 129:813-828

257. Torabi-Parizi P, Davey RT Jr, Suffredini AF, Chertow DS (2015) Ethical and practical considerations in providing critical care to patients with ebola virus disease. Chest 147:1460-1466

258. Blenkharn J, Buckingham SE, Zideman DA (1990) Prevention of transmission of infection during mouth-to-mouth resuscitation. Resuscitation 19:151-157

259. Cydulka RK, Connor PJ, Myers TF, Pavza G, Parker M (1991) Prevention of oral bacterial flora transmission by using mouth-to-mask ventilation during CPR. JEmerg Med 9:317-321

260. Adelborg K, Bjornshave K, Mortensen MB, Espeseth E, WolffA, Lofgren B (2014) A randomised crossover comparison of mouth-to-face-shield ventilation and mouth-to-pocket-mask ventilation by surf lifeguards in a manikin. Anaesthesia 69:712-716

261. Adelborg K, Dalgas C, Grove EL, Jorgensen C, Al-Mashhadi RH, Lofgren B (2011) Mouth-tomouth ventilation is superior to mouth-to-pocket mask and bag-valve-mask ventilation during lifeguard CPR: a randomized study. Resuscitation 82:618-622

262. Paal P, Falk M, Sumann $G$ et al (2006) Comparison of mouth-to-mouth, mouth-to-mask and mouth-to-face-shield ventilation by lay persons. Resuscitation 70:117-123

263. Fingerhut LA, Cox CS, Warner M (1998) International comparative analysis of injury mortality. Findings from the ICE on injury statistics. International Collaborative Effort on Injury Statistics. Adv Data 1998:1-20
264. Wong SC, Tariq SM (2011) Cardiac arrest following foreign-body aspiration. Respir Care 56:527-529

265. Proceedings of the 2005 International Consensus on Cardiopulmonary Resuscitation and Emergency Cardiovascular Care Science with Treatment Recommendations. Resuscitation (2005) 67:157-341

266. Redding JS (1979) The choking controversy: critique of evidence on the Heimlich maneuver. Crit Care Med 7:475-479

267. Langhelle A, Sunde K, WikL, Steen PA (2000) Airway pressure with chest compressions versus Heimlich manoeuvre in recently dead adults with complete airway obstruction. Resuscitation 44:105-108

268. Guildner CW, Williams D, Subitch T (1976) Airway obstructed by foreign material: the Heimlich maneuver. JACEP 5:675-677

269. Ruben $\mathrm{H}$, Macnaughton FI (1978) The treatment of food-choking. Practitioner 221:725-729

270. Kinoshita K, Azuhata T, Kawano D, Kawahara Y (2015) Relationships between pre-hospital characteristics and outcome in victims of foreign body airway obstruction during meals. Resuscitation 88:63-67 